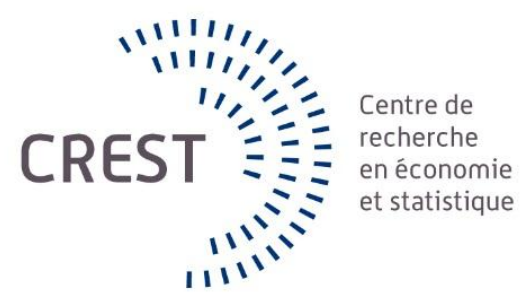

Série des Documents de Travail

\title{
$n^{\circ}$ 2014-40
}

\section{Adaptive Deconvolution on the Nonnegative Real Line}

\author{
Gwennaëlle. MABON ${ }^{1}$
}

Les documents de travail ne reflètent pas la position du CREST et n'engagent que leurs auteurs. Working papers do not reflect the position of CREST but only the views of the authors. 


\title{
ADAPTIVE DECONVOLUTION ON THE NONNEGATIVE REAL LINE
}

\author{
GWENNAËLLE MABON \\ CREST - ENSAE \\ 3 avenue Pierre Larousse \\ 92245 Malakoff, France \\ MAP5, Université Paris Descartes \\ 45 rue des Saints-Pères \\ 75006 Paris, France
}

\begin{abstract}
In this paper we consider the problem of adaptive density or survival function estimation in an additive model defined by $Z=X+Y$ with $X$ independent of $Y$, when both random variables are nonnegative. We want to recover the distribution of $X$ (density or survival function) through $n$ observations of $Z$, assuming that the distribution of $Y$ is known. This issue can be seen as the classical statistical problem of deconvolution which has been tackled in many cases using Fourier-type approaches. Nonetheless, in the present case the random variables have the particularity to be $\mathbb{R}^{+}$supported. Knowing that, we propose a new angle of attack by building a projection estimator with an appropriate Laguerre basis. We present upper bounds on the mean squared integrated risk of our density and survival function estimators. We then describe a nonparametric adaptive strategy for selecting a relevant projection space. The procedures are illustrated with simulated data and compared to the performances of more classical deconvolution setting using a Fourier approach.
\end{abstract}

Keywords. Inverse problem. Adaptive estimation. Nonparametric density estimation. Survival function estimation. Laguerre basis. Deconvolution. Mean squared risk.

AMS Subject Classification 2010: 62G05, 62G07, 62G99, 62N99.

\section{INTRODUCTION}

In this paper we consider the following model

$$
Z_{i}=X_{i}+Y_{i}, \quad i=1, \ldots, n,
$$

where the $X_{i}$ 's are independent identically distributed (i.i.d.) nonnegative variables with unknown density $f$ and unknown survival function $S_{X}$. The $Y_{i}$ 's are also i.i.d. nonnegative variables with known density $g$ and survival function $S_{Y}$. We denote by $h$ the density of the $Z_{i}$ 's and $S_{Z}$ its survival function. Moreover the $X_{i}$ 's and the $Y_{i}$ 's are assumed to be independent. Our target is the estimation of the density $f$ along with the survival function $S_{X}$ of the $X_{i}$ 's when the $Z_{i}$ 's are observed. We are going to show that the assumption of nonnegativity of the random variables is of huge importance for the estimation strategy.

The assumptions imply that, in Model $(1), h(x)=(f * g)(x)$ where $(\varphi * \psi)(x)=\int \varphi(x-u) \psi(u) \mathrm{d} u$ denotes the convolution product. This setting matches the setting of convolution model which is classical in nonparametric statistics. Indeed the problem of recovering the signal distribution $f$ when it is observed with an additive noise with known error distribution, has been extensively studied. We can cite Carroll and Hall (1988), Stefanski (1990), Stefanski and Carroll (1990), Fan (1991), Efromovich (1997) and Delaigle and Gijbels (2004) who study rates of convergence and their optimality for kernel estimators or Butucea (2004), Butucea and Tsybakov (2008a,b) for studies of rate optimality in the minimax sense. For the most part, the adaptive bandwidth selection in deconvolution models has been addressed with a known error distribution, see for example Pensky and Vidakovic (1999) for wavelet strategy, Comte et al. (2006), for projection strategies with penalization, or Meister (2009) and references therein. More recently deconvolution problems in additive models in the case of unknown error distribution have been addressed. For that some information on the error distribution is required. For instance, in a physical context, a preliminary sample of the noise can be derived. This led to the works of Neumann (1997) who proposed an estimation strategy still based

E-mail address: gwennaelle.mabon@ensae.fr.

Date: October 23, 2014. 
on Fourier inversion, or Johannes (2009), Comte and Lacour (2011) and Kappus and Mabon (2013) who extended it to the adaptive strategy. Concerning the estimation of the cumulative distribution function (c.d.f.) in the convolution model, very few papers can be found as Zhang (1990), Fan (1991), Hall and Lahiri (2008), Dattner et al. (2011), Dattner and Reiser (2013). They all present pointwise estimation procedures since the distribution function is not squared integrable on $\mathbb{R}$ while the assumption is not so strong for the survival function on $\mathbb{R}^{+}$. The last two papers consider the pointwise estimation of the c.d.f. when the error distribution is unknown under the assumption that the tail of the characteristic function of the measurement error distribution has a certain decay: polynomially or exponentially. These estimators reach the optimal rates under the conditions that the target function belongs to a Sobolev space.

All these works suppose that the variables $X_{i}$ 's and $Y_{i}$ 's are $\mathbb{R}$-supported. Therefore they are still valid when the variables are $\mathbb{R}^{+}$-supported. Nonetheless in the present paper, our goal is to propose a specific solution for nonnegative variables. We shall illustrate that in practice our procedure works significantly better than Fourier methods for estimating $\mathbb{R}^{+}$-supported functions.

The problem of nonnegative variables appears in actuarial or insurance models. Recently, in a financial context, some papers as Jirak et al. (2014) or Reiß and Selk (2013) have addressed the problem of one-sided errors. The first authors are interested in the optimal adaptive estimation in nonparametric regression when the errors are not assumed to be centered anymore, and typically with Exponential density. It is motivated from fields where the information provided about the error distribution is its support rather than its mean properties. Such matters arise in economics: for example in auction fields the underlying distribution of bidders' private values is identified from observed bids, see Guerre et al. (2000). This led Jirak et al. (2014) to a different approach based on local extreme values. We can also cite Bibinger et al. (2014) who are motivated by modelizing the dynamics of intra-day financial data from limit order books. In all these works one-sided errors models require new and different tools. More generally, the field of survival data analysis and reliability is widespread in many domains such as econometrics or biology, and they also involve nonnegative variables. Duration models are used as soon as the phenomenon of interest is modelized by nonnegative variables which generally corresponds to a waiting time until the occurrence of a certain event such as a failure time. Model (1) can thus be seen as a superposition of two such processes.

Let us describe now our specific method for the estimation of the density and survival functions when the random variables $X$ and $Y$ in Model (1) are $\mathbb{R}^{+}$-supported. We assume all along the paper that $g$ belongs to $\mathbb{L}^{2}\left(\mathbb{R}^{+}\right)$and either

(A1) $f \in \mathbb{L}^{2}\left(\mathbb{R}^{+}\right)$

when the estimation of $f$ is under study, or

(B1) $S_{X} \in \mathbb{L}^{2}\left(\mathbb{R}^{+}\right)$,

when we want to recover the survival function. In both cases, we use a penalized projection method (see Birgé and Massart (1997)). The idea is to decompose the density function $f$ on an appropriate orthonormal basis on $\mathbb{R}^{+},\left(\varphi_{k}\right)_{k \geq 0}$,

$$
f(x)=\sum_{k \geq 0} b_{k}(f) \varphi_{k}(x)
$$

where $b_{k}(f)$ represents the $k$-th component of $f$ in the orthonormal basis and to estimate the $m$ first ones $b_{0}(f), \ldots, b_{m-1}(f)$. To deal with the particularity of nonnegative variables we introduce the Laguerre basis defined by

$$
k \in \mathbb{N}, x \geq 0, \quad \varphi_{k}(x)=\sqrt{2} L_{k}(2 x) e^{-x} \quad \text { with } \quad L_{k}(x)=\sum_{j=0}^{k}(-1)^{j}\left(\begin{array}{l}
k \\
j
\end{array}\right) \frac{x^{j}}{j !} .
$$

This basis has already been used to estimate a nonnegative function $f$ in Comte et al. (2013). These authors consider a regression model defined by $Y_{i}=f * g\left(t_{i}\right)+\varepsilon_{i}$ where $Y_{i}$ is observed, $t_{i}$ are deterministic times of observation, $\varepsilon_{i}$ is Gaussian and $g$ is known. We can also cite Vareschi (2014) in a similar context with unknown $g$. For $\mathbb{R}^{+}$-supported functions, the convolution product writes

$$
h(x)=\int_{0}^{x} f(u) g(x-u) \mathrm{d} u
$$

and what makes the Laguerre basis relevant, in the previous works and in ours, is the relation

$$
\int_{0}^{x} \varphi_{k}(u) \varphi_{j}(x-u) \mathrm{d} u=2^{-1 / 2}\left(\varphi_{k+j}(x)-\varphi_{k+j+1}(x)\right) .
$$

(see formula 22.13.14 in Abramowitz and Stegun (1964)). From this property, by decomposing $f, g$ and $h$ on the Laguerre basis, we are able to define a linear transformation of the coefficients of the density function 
$f$ to obtain those of $h$. More precisely, if we denote by $\vec{h}_{m}$ and $\vec{f}_{m} m$-dimensional vectors with coordinates $b_{k}(f)$ and $b_{k}(h), k=0,1, \ldots, m-1$ respectively, we prove

$$
\vec{h}_{m}=\mathbf{G}_{m} \vec{f}_{m}
$$

where $\mathbf{G}_{m}$ is a lower triangular invertible matrix depending on the coefficients of $g$. As $g$ is known, so is $\mathbf{G}_{m}$. Thus we can recover the $m$ first coefficients of $f$, from those of $g$ which are known and those of $h$ which can be estimated form the $Z_{i}$ 's as $b_{k}(h)=\mathbb{E}\left[\varphi_{k}\left(Z_{1}\right)\right]$. We then derive the same reasoning for the survival function estimation. Let us point out that we do not integrate the estimator by projection of $f$ to obtain an estimator of $S_{X}$. Our idea is to directly project $S_{X}$ on the Laguerre basis. To our knowledge this a new strategy for the survival function estimation in a deconvolution setting. These estimators are precisely defined and illustrated in Section 2.

Afterwards we develop in Section 3 a study of the integrated risk of the estimator of the density and of the survival function. We discuss the resulting rates of convergence of these two estimators. For that we introduce subspaces of $\mathbb{L}^{2}\left(\mathbb{R}^{+}\right)$, called Laguerre-Sobolev spaces with index $s>0$ which are defined in Bongioanni and Torrea (2009). This enables us to determine the order of the squared bias terms. This, together with variance order, provides rates of convergence of the estimators of $f$ belonging to a LaguerreSobolev space. We also obtain rates of convergence for estimators of survival function.

In Section 4, we establish a data driven choice by penalization of the dimension $m$ in our two models and oracle inequalities. For the estimation of the density and survival functions, the methods rely mostly on the fact that we are able to build nested models since the first $m-1$ coordinates $\vec{h}_{m}$ and $\vec{f}_{m}$ are the same as those of $\vec{h}_{m-1}$ and $\vec{f}_{m-1}$. Finally we illustrate these procedures with some simulations and compare our results to those of Comte et al. (2006) in the case of the density estimation.

To sum up this paper is organized as follows. In Section 2, we give the notations, specify the statistical model and estimation procedures for $f$ and $S_{X}$. In Section 3, we present upper bounds of the $\mathbb{L}^{2}$ integrated risk and derive the corresponding rates of convergence. In Section 4, we propose a new adaptive procedure by penalization for the density and survival functions. Besides the theoretical properties of the adaptive estimators are studied. In Section 5, we lead a study of the adaptive estimators through simulation experiments. Numerical results are then presented and compared to the performances in a more classical deconvolution setting using a Fourier approach. In the concluding Section 6 we give further possible developments or extensions of the method. All the proofs are postponed to Section 7.

\section{Statistical MOdel AND EStimation Procedure}

2.1. Notations. For two real numbers $a$ and $b$, we denote $a \vee b=\max (a, b)$ and $a \wedge b=\min (a, b)$. For two functions $\varphi, \psi: \mathbb{R} \rightarrow \mathbb{R}$ belonging to $\mathbb{L}^{1}(\mathbb{R}) \cap \mathbb{L}^{2}(\mathbb{R})$, we denote $\|\varphi\|$ the $\mathbb{L}^{2}$ norm of $\varphi$ defined by $\|\varphi\|^{2}=\int_{\mathbb{R}}|\varphi(x)|^{2} d x,\langle\varphi, \psi\rangle$ the scalar product between $\varphi$ and $\psi$ defined by $\langle\varphi, \psi\rangle=\int_{\mathbb{R}} \varphi(x) \overline{\psi(x)} d x$.

Let $d$ be an integer, for two vectors $\vec{u}$ and $\vec{v}$ belonging to $\mathbb{R}^{d}$, we denote $\|\vec{u}\|_{2, d}$ the Euclidean norm defined by $\|\vec{u}\|_{2, d}^{2}={ }^{t} \vec{u} \vec{u}$ where ${ }^{t} \vec{u}$ is the transpose of $\vec{u}$. The scalar product between $\vec{u}$ and $\vec{v}$ is $\langle\vec{u}, \vec{v}\rangle_{2, d}=\sqrt{{ }^{t} \vec{u} \vec{v}}=$ $\sqrt{{ }^{t} \vec{v} \vec{u}}$. We introduce the spectral norm of a matrix $\mathbf{A}: \varrho^{2}(\mathbf{A})=\lambda_{\max }\left({ }^{t} \mathbf{A} \mathbf{A}\right)$ where $\lambda_{\max }(\mathbf{A})$ is the largest eigenvalue of $A$ in absolute value.

2.2. Laguerre basis. The Laguerre polynomials $L_{k}$ defined by (2) are orthonormal with respect to the weight function $x \mapsto e^{-x}$ on $\mathbb{R}^{+}$. In other words, $\int_{\mathbb{R}^{+}} L_{k}(x) L_{k^{\prime}}(x) e^{-x} \mathrm{~d} x=\delta_{k, k^{\prime}}$ where $\delta_{k, k^{\prime}}$ is the Kronecker symbol. We remind that the Laguerre basis verifies the following inequality for all integer $k$

$$
\sup _{x \in \mathbb{R}^{+}}\left|\varphi_{k}(x)\right|=\left\|\varphi_{k}\right\|_{\infty} \leq \sqrt{2} .
$$

We also introduce the space $\mathcal{S}_{m}=\operatorname{Span}\left\{\varphi_{0}, \ldots, \varphi_{m-1}\right\}$.

2.3. Projection estimator of the density function. For a function $p$ in $\mathbb{L}^{2}\left(\mathbb{R}^{+}\right)$, we note

$$
p(x)=\sum_{k \geq 0} b_{k}(p) \varphi_{k}(x) \quad \text { where } \quad b_{k}(p)=\int_{\mathbb{R}^{+}} p(u) \varphi_{k}(u) \mathrm{d} u .
$$

Thus under Assumption (A1), $f$ and $g$ admit a development on the Laguerre basis. Since $X$ and $Y$ are independent and nonnegative variables, we have a convolution relation between $h, f$ and $g$. Starting from Equation (3) and using the decomposition on the Laguerre basis, the following equality holds

$$
h(x)=\sum_{k=0}^{\infty} \sum_{j=0}^{\infty} b_{k}(f) b_{j}(g) \int_{0}^{x} \varphi_{k}(u) \varphi_{j}(x-u) \mathrm{d} u .
$$


So we decompose $h$ on the Laguerre basis as $\sum_{k=0}^{\infty} b_{k}(h) \varphi_{k}(x)$ and apply Equation (4) to (7). We get

$$
\sum_{k=0}^{\infty} b_{k}(h) \varphi_{k}(x)=\sum_{k=0}^{\infty} \varphi_{k}(x)\left(2^{-1 / 2} b_{k}(f) b_{0}(g)+\sum_{l=0}^{k-1} 2^{-1 / 2}\left(b_{k-l}(g)-b_{k-l-1}(g)\right) b_{l}(f)\right) .
$$

We finally obtain an infinite triangular system of linear equations. We can write for any $m$ that $\vec{h}_{m}=\mathbf{G}_{m} \vec{f}_{m}$ where $\mathbf{G}_{m}$ is the lower triangular Toeplitz matrix with elements

$$
\mathbf{G}_{m}= \begin{cases}2^{-1 / 2} b_{0}(g) & \text { if } i=j, \\ 2^{-1 / 2}\left(b_{i-j}(g)-b_{i-j-1}(g)\right) & \text { if } j<i, \\ 0 & \text { otherwise }\end{cases}
$$

(see Comte et al. (2013)). Let us give some examples of matrix $\mathbf{G}_{m}$.

Example 1. If $g$ is an Exponential distribution of parameter $\theta>0$, then for all integer $k$ we have

$$
\begin{aligned}
b_{k}(g) & =\int_{\mathbb{R}^{+}} g(u) \varphi_{k}(u) \mathrm{d} u=\int_{\mathbb{R}^{+}} \theta e^{-\theta u} \sqrt{2} e^{-u} L_{k}(2 u) \mathrm{d} u=\sqrt{2} \theta \sum_{j=0}^{k}\left(\begin{array}{l}
k \\
j
\end{array}\right) \frac{(-1)^{j}}{j !} \int_{\mathbb{R}^{+}}(2 u)^{j} e^{-(1+\theta) u} \mathrm{~d} u \\
& =\frac{\sqrt{2} \theta}{\theta+1} \sum_{j=0}^{k}\left(\begin{array}{l}
k \\
j
\end{array}\right) \frac{(-2)^{j}}{(1+\theta)^{j}}=\frac{\sqrt{2} \theta}{\theta+1} \frac{(\theta-1)^{k}}{(\theta+1)^{k}} .
\end{aligned}
$$

Then we have that

$$
b_{0}(g)=\frac{\sqrt{2} \theta}{1+\theta} \quad \text { and } \quad b_{i-j}(g)-b_{i-j-1}(g)=-2 \sqrt{2} \theta \frac{(\theta-1)^{i-j-1}}{(\theta+1)^{i-j+1}} .
$$

Thus in a case of an Exponential error distribution, the coefficients of $\mathbf{G}_{m}$ can easily be computed. Nonetheless in the next example, we show that for a Gamma distribution the coefficients can also be recovered.

Example 2. If $g$ is a Gamma distribution of parameters $p \in \mathbb{N}^{*}$ and $\theta>0$, then for all integer $k$ we have

$$
\begin{aligned}
b_{k}(g) & =\int_{\mathbb{R}^{+}} g(u) \varphi_{k}(u) \mathrm{d} u=\int_{\mathbb{R}^{+}} \theta^{p} u^{p-1} e^{-\theta u} \sqrt{2} e^{-u} L_{k}(2 u) \mathrm{d} u=\sqrt{2} \theta^{p} \sum_{j=0}^{k}\left(\begin{array}{c}
k \\
j
\end{array}\right) \frac{(-1)^{j}}{j !} 2^{j} \int_{\mathbb{R}^{+}} u^{p+j-1} e^{-(1+\theta) u} \mathrm{~d} u \\
& =\sqrt{2} \frac{\theta^{p}}{(1+\theta)^{p}} \sum_{j=0}^{k}\left(\begin{array}{l}
k \\
j
\end{array}\right)(-1)^{j} \frac{2^{j}}{(1+\theta)^{j}} \frac{(p+j-1) !}{j !}=\left.\frac{\sqrt{2} \theta^{p}}{(1+\theta)^{p}} \frac{\mathrm{d}^{p-1}}{\mathrm{~d} x^{p-1}}\left[x^{p-1}(1-x)^{k}\right]\right|_{x=2 /(1+\theta)}
\end{aligned}
$$

The principle of a projection method for estimation is to reduce the question of estimating $f$ to the one of estimating $f_{m}$ the projection of $f$ on $\mathcal{S}_{m}$. Clearly

$$
f_{m}(x)=\sum_{k=0}^{m-1} b_{k}(f) \varphi_{k}(x)
$$

and $b_{k}(f)$ for $k=0, \ldots, m-1$ can be estimated by Equation (5) if we can invert the matrix $\mathbf{G}_{m}$. Since $\mathbf{G}_{m}$ is a lower triangular matrix, it is invertible only if the coefficients of the diagonal are different from 0 which means $b_{0}(g)$ is non equal to 0 . Well by definition we have

$$
b_{0}(g)=\int_{\mathbb{R}^{+}} g(u) \varphi_{0}(u) \mathrm{d} u=\sqrt{2} \int_{\mathbb{R}^{+}} g(u) e^{-u} \mathrm{~d} u=\sqrt{2} \mathbb{E}\left[e^{-Y}\right]>0 .
$$

Consequently $\mathbf{G}_{m}$ is invertible. Then we can write that $\mathbf{G}_{m}^{-1} \vec{h}_{m}=\vec{f}_{m}$. So, as $b_{k}(h)=\mathbb{E}\left[\varphi_{k}\left(Z_{1}\right)\right]$, the projection of $f$ on $\mathcal{S}_{m}$ can be estimated by

$$
\hat{f}_{m}(x)=\sum_{k=0}^{m-1} \hat{b}_{k}(f) \varphi_{k}(x) \quad \text { with } \quad \hat{\vec{f}}_{m}=\mathbf{G}_{m}^{-1} \hat{\vec{h}}_{m} \quad \text { and } \quad \hat{b}_{k}(h)=\frac{1}{n} \sum_{i=1}^{n} \varphi_{k}\left(Z_{i}\right) .
$$

Let us notice that if $Y=0$ a.s. then $g=\delta_{0}$ for all integer $k$, and we have $b_{k}(g)=\varphi_{k}(0)=\sqrt{2}$ because $L_{k}(0)=1$. This implies $\mathbf{G}_{m}=\operatorname{Id}_{m}$, with $\operatorname{Id}_{m}$ the identity matrix. Therefore if there is no additional noise, we are able to estimate $f_{m}$ directly from the observations. It means that in Equation (9) we have

$$
\mathbf{G}_{m}=\operatorname{Id}_{m} \quad \text { and } \quad \hat{\vec{f}}_{m}=\hat{\vec{h}}_{m}
$$


2.4. Projection estimator of the survival function. We have to point out that in the case of the projection estimation of the survival function, the estimation of the coefficients in the Laguerre basis is slightly different from the previous section. Indeed we note that the scalar product cannot be interpreted directly as an expectation under $h$. Let us consider for example $b_{k}\left(S_{Z}\right)$ the $k$-th coefficient of $S_{Z}$

$$
b_{k}\left(S_{Z}\right)=\int_{\mathbb{R}^{+}} S_{Z}(u) \varphi_{k}(u) \mathrm{d} u=\int_{\mathbb{R}^{+}} \varphi_{k}(u)\left(\int_{u}^{+\infty} h(v) \mathrm{d} v\right) \mathrm{d} u=\int_{\mathbb{R}^{+}}\left(\int_{0}^{v} \varphi_{k}(u) \mathrm{d} u\right) h(v) \mathrm{d} v=\mathbb{E}\left[\Phi_{k}\left(Z_{1}\right)\right]
$$

with $\Phi_{k}$ a primitive of $\varphi_{k}$ defined as $\Phi_{k}(x)=\int_{0}^{x} \varphi_{k}(u) \mathrm{d} u$. We can notice that

$$
\Phi_{k}(x)=\sqrt{2} \int_{0}^{x} \sum_{j=0}^{k}(-2)^{j}\left(\begin{array}{l}
k \\
j
\end{array}\right) \frac{u^{j}}{j !} e^{-u} \mathrm{~d} u=\sqrt{2} \sum_{j=0}^{k}(-2)^{j}\left(\begin{array}{l}
k \\
j
\end{array}\right) \gamma(j+1, x) .
$$

where $\gamma$ is the lower incomplete gamma function defined by formula 6.5.2. in Abramowitz and Stegun (1964). In order to apply the same method as for the density estimation, let us see how convolution is modified for survival functions. Let $z \geq 0$, by definition $S_{Z}(z)=\mathbb{P}(Z>z)$, and

$$
\begin{aligned}
S_{Z}(z) & =\mathbb{P}(X+Y>z)=\iint \mathbb{1}_{x+y>z} f(x) \mathbb{1}_{x \geq 0} g(y) \mathbb{1}_{y \geq 0} \mathrm{~d} x \mathrm{~d} y \\
& =\int\left(\int_{z-y}^{+\infty} f(x) \mathrm{d} x\right) g(y) \mathbb{1}_{y \geq 0} \mathbb{1}_{z-y \geq 0} \mathrm{~d} y+\int\left(\int_{0}^{+\infty} f(x) \mathrm{d} x\right) g(y) \mathbb{1}_{y \geq 0} \mathbb{1}_{z-y \leq 0} \mathrm{~d} y \\
& =\int_{0}^{z} S_{X}(z-y) g(y) \mathrm{d} y+\int_{z}^{+\infty} g(y) \mathrm{d} y \\
& =\int_{0}^{z} S_{X}(z-y) g(y) \mathrm{d} y+S_{Y}(z) .
\end{aligned}
$$

We can notice that we have one more term: the survival function of $Y$. Nevertheless similarly to the density estimation the coefficients of $S_{X}, b_{k}\left(S_{X}\right)$ can also be represented as a solution of an infinite triangular system of linear equations as follows

$$
\begin{aligned}
S_{Z}(z)-S_{Y}(z) & =\sum_{k \geq 0}\left(b_{k}\left(S_{Z}\right)-b_{k}\left(S_{Y}\right)\right) \varphi_{k}(z) \\
& =2^{-1 / 2} \sum_{k=0}^{\infty} \varphi_{k}(x)\left(b_{k}\left(S_{X}\right) b_{0}(g)+\sum_{l=0}^{k}\left(b_{(k-l)}(g)-b_{(k-l-1)}(g)\right) b_{l}\left(S_{X}\right)\right) .
\end{aligned}
$$

Now let us define, $S_{X, m}$ the projection of $S_{X}$ on the space $\mathcal{S}_{m}$

$$
S_{X, m}(x)=\sum_{k=0}^{m-1} b_{k}\left(S_{X}\right) \varphi_{k}(x) .
$$

Thus, with $\mathbf{G}_{m}$ defined by Equation (8) and $\Phi_{k}$ defined by (11), the projection estimator of $S_{X, m}$ on the Laguerre basis is given by

$$
\begin{gathered}
\widehat{S}_{X, m}(x)=\sum_{k=0}^{m-1}\left(\hat{b}_{k}\left(S_{Z}\right)-b_{k}\left(S_{Y}\right)\right) \varphi_{k}(x) \\
\text { with } \widehat{\vec{S}}_{X, m}=\mathbf{G}_{m}^{-1}\left(\widehat{\vec{S}}_{Z, m}-\vec{S}_{Y, m}\right) \text { and } \hat{b}_{k}\left(S_{Z}\right)=\frac{1}{n} \sum_{i=1}^{n} \Phi_{k}\left(Z_{i}\right),
\end{gathered}
$$

where $\vec{S}_{Y, m}$ is known since $b_{k}\left(S_{Y}\right)=\mathbb{E}\left[\Phi_{k}\left(Y_{1}\right)\right]$ and $g$ is known.

It is worth mentioning that here we do not integrate the estimator of the density $\hat{f}_{m}$ to estimate the survival function. Otherwise the estimator of the survival function at a point $x$ would be expressed in function of the coefficients $\hat{\vec{f}}_{m}$ and first antiderivative of the $\varphi_{k}$ noted $\tilde{\Phi}_{k}$ as follows

$$
\widetilde{S}_{X, m}(x)=\sum_{k=0}^{m-1} \hat{b}_{k}(f) \tilde{\Phi}_{k}(x)
$$

with $\tilde{\Phi}_{k}(x)=\int_{x}^{+\infty} \varphi_{k}(u) \mathrm{d} u$. Yet in order to evaluate the bias and the variance, we would rather have a decomposition directly on the Laguerre basis as $\widetilde{S}_{X, m}(x)=\sum_{k=0}^{m-1} \tilde{b}_{k}(f) \varphi_{k}(x)$. This part becomes tedious, see Appendix B for more details. 
Moreover we want to highlight that this method would require $f \in \mathbb{L}^{2}\left(\mathbb{R}^{+}\right)$. Yet it possible that $S_{X} \in$ $\mathbb{L}^{2}\left(\mathbb{R}^{+}\right)$and $f \notin \mathbb{L}^{2}\left(\mathbb{R}^{+}\right)$. For instance let us define $f(x)=1 /(2 \sqrt{x}) \mathbb{1}_{[0,1]}(x), f \notin \mathbb{L}^{2}\left(\mathbb{R}^{+}\right)$and $S_{X} \in \mathbb{L}^{2}\left(\mathbb{R}^{+}\right)$. Now if we take $f(x)=2 x^{-3 / 2} \mathbb{1}_{[1,+\infty)}(x)$ then $S(x)=4 x^{-1 / 2} \mathbb{1}_{[1,+\infty)}(x)$ which implies $f \in \mathbb{L}^{2}\left(\mathbb{R}^{+}\right)$and $S_{X} \notin \mathbb{L}^{2}\left(\mathbb{R}^{+}\right)$.

\section{BOUndS ON THE $\mathbb{L}^{2}$ RISK}

In this section, we study the integrated risk of our three estimators. We remind that

$$
\mathbb{E}\left\|f-\hat{f}_{m}\right\|^{2}=\underbrace{\left\|f-f_{m}\right\|^{2}}_{\text {squared bias }}+\underbrace{\mathbb{E}\left\|f_{m}-\hat{f}_{m}\right\|^{2}}_{\text {variance }} .
$$

First we give upper bounds of the $\mathbb{L}^{2}$ risk by bounding the variance term then we compute optimal rates by introducing the Laguerre-Sobolev space and estimating the order of the bound on the variance for each estimators.

\subsection{Upper bounds.}

Proposition 3.1. Under (A1), for $\mathbf{G}_{m}$ defined by (8) and $\hat{f}_{m}$ defined by (9), the following result holds

$$
\mathbb{E}\left\|f-\hat{f}_{m}\right\|^{2} \leq\left\|f-f_{m}\right\|^{2}+\frac{2 m}{n} \varrho^{2}\left(\mathbf{G}_{m}^{-1}\right) .
$$

This result can easily be applied to the direct estimation of the density as stated

Corollary 3.2. Under (A1), in the forward problem defined by (10) we get

$$
\mathbb{E}\left\|f-\hat{f}_{m}\right\|^{2} \leq\left\|f-f_{m}\right\|^{2}+\frac{2 m}{n} .
$$

Finally, we derive the following upper bound for the projection estimator of the survival function.

Proposition 3.3. Under (B1) and if $\mathbb{E}\left[Z_{1}\right]<\infty$, for $\mathbf{G}_{m}$ defined by (8) and $\widehat{S}_{X, m}$ defined by (13), the following result holds

$$
\mathbb{E}\left\|S_{X}-\widehat{S}_{X, m}\right\|^{2} \leq\left\|S_{X}-S_{X, m}\right\|^{2}+\frac{\mathbb{E}\left[Z_{1}\right]}{n} \varrho^{2}\left(\mathbf{G}_{m}^{-1}\right)
$$

Lemma 3.4. $m \mapsto \varrho^{2}\left(\mathbf{G}_{m}^{-1}\right)$ is nondecreasing.

Remark 1. The terms of the right-hand side of Equations (14), (15) and (16) correspond to a squared bias and variance term. Indeed the first one gets smaller when $m$ gets larger and vice versa for the other one thanks to Lemma 3.4.

3.2. Rates of convergence. In order to derive the corresponding rates of convergence of the estimators $\hat{f}_{m}$ and $\widehat{S}_{X, m}$ respectively defined by (9) and (13), we need to evaluate the smoothness of the signal along with the order of $\varrho^{2}\left(\mathbf{G}_{m}^{-1}\right)$. In the first place, we assume that $f$ belongs to a Laguerre-Sobolev space defined as

$$
W^{s}\left(\mathbb{R}^{+}, L\right)=\left\{f: \mathbb{R}^{+} \rightarrow \mathbb{R}, f \in \mathbb{L}^{2}\left(\mathbb{R}^{+}\right), \sum_{k \geq 0} k^{s} b_{k}^{2}(f) \leq L<+\infty\right\} \quad \text { with } \quad s \geq 0
$$

where $b_{k}(f)=\left\langle f, \varphi_{k}\right\rangle$. Bongioanni and Torrea (2009) have introduced Laguerre-Sobolev space but the link with the coefficients of a function on a Laguerre basis was done by Comte and Genon-Catalot (2013). Indeed, let $s$ be an integer, for $f: \mathbb{R}^{+} \rightarrow \mathbb{R}$ and $f \in \mathbb{L}^{2}\left(\mathbb{R}^{+}\right)$, we have that

$$
\sum_{k \geq 0} k^{s} b_{k}^{2}(f)<+\infty
$$

is equivalent to the fact that $f$ admits derivatives up to order $s-1$ with $f^{(s-1)}$ absolutely continuous and for $0 \leq k \leq s, x^{k / 2} f^{(k)}(x) e^{-x} \in \mathbb{L}^{2}\left(\mathbb{R}^{+}\right)$. For more details we refer to section 7 of Comte and Genon-Catalot (2013). Now for $f \in W^{s}\left(\mathbb{R}^{+}, L\right)$ defined by (17),

$$
\left\|f-f_{m}\right\|^{2}=\sum_{k=m}^{\infty} b_{k}^{2}(f)=\sum_{k=m}^{\infty} b_{k}^{2}(f) k^{s} k^{-s} \leq L m^{-s} .
$$

Before deriving the order of the spectral norm of $\mathbf{G}_{m}^{-1}$, we can already give the rate of convergence in the forward problem. 
Proposition 3.5. In the forward problem defined by $(10)$, suppose that $f$ belongs to $W^{s}\left(\mathbb{R}^{+}, L\right)$ defined by (17) and let $m_{\text {opt }}=(2 / s L)^{-1 /(s+1)} n^{1 /(s+1)}$, then the following holds

$$
\sup _{f \in W^{s}\left(\mathbb{R}^{+}, L\right)} \mathbb{E}\left\|f-\hat{f}_{m_{\text {opt }}}\right\|^{2} \leq C_{1}(s, L) n^{-s /(s+1)}
$$

where $C_{1}(s, L)=L(2 / s L)^{-s /(s+1)}+2(2 / s L)^{-1 /(s+1)}$.

Secondly in the deconvolution problem, we must evaluate the variance term of Equations (14) and (16) which means assess the order of $\varrho^{2}\left(\mathbf{G}_{m}^{-1}\right)$. Comte et al. (2013) show that under the following conditions on the density $g$, we can recover the order of the spectral norm of $\mathbf{G}_{m}^{-1}$ :

(C1) $g \in \mathbb{L}^{1}\left(\mathbb{R}^{+}\right)$is $r$ times differentiable and $g^{(r)} \in \mathbb{L}^{1}\left(\mathbb{R}^{+}\right)$.

(C2) There exists an integer $r \geq 1$ such that

$$
\left.\frac{\mathrm{d}^{j}}{\mathrm{~d} x^{j}} g(x)\right|_{x=0}= \begin{cases}0 & \text { if } j=0,1, \ldots, r-2 \\ B_{r} \neq 0 & \text { if } j=r-1 .\end{cases}
$$

(C3) The Laplace transform of $g$ has no zero with non negative real parts except for the zeros of the form $\infty+i b$.

Lemma 3.6 (Comte et al. (2013)). If Assumptions (C1)-(C3) are true, then there exists a positive constant $C_{\varrho}$ such that

$$
\varrho^{2}\left(\mathbf{G}_{m}^{-1}\right) \leq C_{\varrho} m^{2 r-1}
$$

Remark 2. For instance a Gamma distribution of parameter $p$ and $\theta$ verifies these three conditions for $r=p$. Especially an Exponential distribution satisfies those assumptions for $r=1$.

Under (C1)-(C3), we consider distributions such that they admit a finite number of derivatives null in 0 .

Proposition 3.7. Assume that $f$ belongs to $W^{s}\left(\mathbb{R}^{+}, L\right)$ defined by (17), that Assumptions (C1)-(C3) are fulfilled and let $m_{\text {opt }}=\left(4 r C_{\varrho} / s L\right)^{-1 /(s+2 r)} n^{1 /(s+2 r)}$, then

$$
\sup _{f \in W^{s}\left(\mathbb{R}^{+}, L\right)} \mathbb{E}\left\|f-\hat{f}_{m_{\text {opt }}}\right\|^{2} \leq C_{2}\left(s, L, C_{\varrho}\right) n^{-s /(s+2 r)} .
$$

where $C_{2}\left(s, L, C_{\varrho}\right)=L\left(4 r C_{\varrho} / s L\right)^{-s /(s+2 r)}+2\left(4 r C_{\varrho} / s L\right)^{-1 /(s+2 r)}$.

Example 3. Let us compute the rate of convergence where both the signal and error distributions are Exponential in the Laguerre procedure and compare it to the rate in the Fourier setting. For an Exponential distribution of parameter $\theta$, we can note that the squared bias decays exponentially. Indeed Example 1 gives the coefficients of an Exponential distribution, thus the squared bias is

$$
\left\|f-f_{m}\right\|^{2}=\sum_{k \geq m}\left(\sqrt{2} \theta \frac{(\theta-1)^{k}}{(\theta+1)^{k+1}}\right)^{2}=\frac{\theta}{2}\left(\frac{\theta-1}{\theta+1}\right)^{2 m} .
$$

From Remark 2, the variance is bounded by $C_{\varrho} m / n$. It implies that if $\theta \neq 1$, the rate of convergence is of order $(\log n) / n$ with $m_{\text {opt }}=2 \log n /|2 \log ((\theta-1) /(\theta+1))|$. It can also be noted that if $\theta=1$ then the bias is null. The rate of convergence, in this case, reaches the parametric rate. In the Fourier deconvolution, in the same Exponential situation for signal and error, the order of the squared bias is $1 / m$ while the order of the variance is $m^{3} / n$. It yields that the rate is $n^{-1 / 4}$. Thus in the context of nonnegative variables of Exponential type, we expect to recover faster rates of convergence with our Laguerre method than with a Fourier procedure.

Proposition 3.8. Assume that $S_{X}$ belongs to $W^{s+1}\left(\mathbb{R}^{+}, L\right)$ defined by (17), that Assumptions (C1)-(C3) are fulfilled and let $m_{\text {opt }}=\left(C_{\varrho} \mathbb{E}\left[Z_{1}\right](2 r-1) /(s+1) L\right)^{-1 /(s+2 r)} n^{1 /(s+2 r)}$, then

$$
\sup _{S_{X} \in W^{s}\left(\mathbb{R}^{+}, L\right)} \mathbb{E}\left\|S_{X}-\widehat{S}_{X, m}\right\|^{2} \leq C_{3}(s, L) n^{-(s+1) /(s+2 r)} .
$$

with $C_{3}(s, L)=L\left(\frac{C_{\varrho} \mathbb{E}\left[Z_{1}\right](2 r-1)}{(s+1) L}\right)^{-(s+1) /(s+2 r)}+2\left(\frac{C_{\varrho} \mathbb{E}\left[Z_{1}\right](2 r-1)}{(s+1) L}\right)^{-1 /(s+2 r)}$

Remark 3. We clearly see that in Propositions 3.5, 3.7 and 3.8 the optimal $m$ that permits to compute the rate of convergence of the estimator depends on the regularity of the function under estimation. So the solution of the best compromise between the squared bias and the variance depends on unknown quantities $L$ and $s$. That is why we consider the problem of data driven selection of $m$. Our goal is then to find a 
procedure that does not require prior information on $f$ nor $S_{X}$ and whose the risk automatically reaches the optimal rate.

Remark 4. Lower bounds in deconvolution problems on the real line have been studied in Fan (1991) and Butucea and Tsybakov (2008a,b), yet those results cannot be extended to the setting of this paper. Therefore the Laguerre deconvolution requires a specific construction. The difficulty lies in the construction of densities on $\mathbb{R}^{+}$. Otherwise we can cite Vareschi (2014) who proves lower bounds in the context of a Laplace regression model.

\section{Model SELECTION AND ADAPTIVE ESTIMATION}

The aim of this section is to provide an integer $m$ that enables us to compute an estimator of the

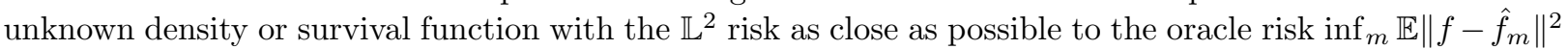
or $\inf _{m} \mathbb{E}\left\|S_{X}-\widehat{S}_{X, m}\right\|^{2}$. We follow the model selection paradigm (see Birgé and Massart (1997), Birgé (1999), Massart (2003)) and choose the dimension of projection spaces $m$ as the minimizer of a penalized criterion.

4.1. Adaptive density estimation. First let us consider the forward problem. We define the following contrast

associated with the penalty

$$
\theta_{n}(t)=\|t\|^{2}-\frac{2}{n} \sum_{i=1}^{n} t\left(X_{i}\right) \quad t \in \mathcal{S}_{m}
$$

$$
\text { qen }(m)=\frac{2 \kappa m}{n}
$$

where $\kappa$ is a numerical constant. The penalty has the classic form of a penalty in model selection as presented in Massart (2003) see formula 7.17 page 212. The following result ensures that the tradeoff between the bias and the variance is automatically reached.

Theorem 4.1. In the forward problem defined by (10), suppose that Assumption (A1) is true. Let $\hat{f}_{\hat{m}}$ be defined by (9) and

$$
\widehat{m}=\underset{m \in\{1, \ldots, n\}}{\operatorname{argmin}}\left\{\theta_{n}\left(\hat{f}_{m}\right)+\mathrm{qen}(m)\right\}
$$

with qen defined by (18), then there exists a positive numerical constant $\kappa$ such that

$$
\mathbb{E}\left\|f-\hat{f}_{\widehat{m}}\right\|^{2} \leq 4 \inf _{m \in\{1, \ldots, n\}}\left\{\left\|f-f_{m}\right\|^{2}+\operatorname{qen}(m)\right\}+\frac{C}{n} .
$$

where $C$ depends on $\|f\|$.

The estimation problem of a density $f$ based on independent observations $X_{1}, \ldots, X_{n}$ has been widely studied in the nonparametric estimation literature, see Silverman (1986) and Devroye (1987). More recently Juditsky and Lambert-Lacroix (2004) have proposed a minimax estimator of the density, based on wavelets decomposition, on the whole real line for the $\mathbb{L}^{p}$-risk $(1 \leq p \leq \infty)$.

Now consider the inverse problem, we add the two following assumptions:

(A2) $\mathcal{M}_{n}^{(1)}=\left\{1 \leq m \leq d_{1}, \frac{m \varrho^{2}\left(\mathbf{G}_{m}^{-1}\right)}{n} \leq 1\right\}$, where $d_{1}<n$ may depend on $n$.

(A3) $\forall b>0, \sum_{m \in \mathcal{M}_{n}^{(1)}} \varrho^{2}\left(\mathbf{G}_{m}^{-1}\right) e^{-b m}<C(b)<\infty$.

Moreover for $m \in \mathcal{M}_{n}^{(1)}$, let us define the associated subspaces $\mathcal{S}_{d_{1}}^{m} \subseteq \mathbb{R}^{d_{1}}$

$$
\mathcal{S}_{d_{1}}^{m}=\left\{\vec{t}_{m} \in \mathbb{R}^{d_{1}} / \vec{t}_{m}={ }^{t}\left(b_{0}(t), b_{1}(t), \ldots, b_{m-1}(t), 0, \ldots, 0\right)\right\} .
$$

This space is defined to give nested models. Thus when we increase the dimension from $m$ to $m+1$ we only compute one more coefficient. Then for any $\vec{t} \in \mathbb{R}^{d_{1}}$, we define the following contrast for the density estimation

$$
\gamma_{n}(\vec{t})=\|\vec{t}\|_{2, d_{1}}^{2}-2\left\langle\vec{t}, \mathbf{G}_{d_{1}}^{-1} \widehat{\vec{h}}_{d_{1}}\right\rangle_{2, d_{1}} .
$$

Let us notice that for $\vec{t}_{m} \in \mathcal{S}_{d_{1}}^{m}$, thanks to the null coordinates of $\vec{t}_{m}$ and the lower triangular form of $\mathbf{G}_{d_{1}}$ and $\mathbf{G}_{m}$, we have

$$
\left\langle\vec{t}_{m}, \mathbf{G}_{d_{1}}^{-1} \widehat{\vec{h}}_{d_{1}}\right\rangle_{2, d_{1}}=\left\langle\vec{t}_{m}, \mathbf{G}_{m}^{-1} \widehat{\vec{h}}_{m}\right\rangle_{2, m}=\left\langle\vec{t}_{m}, \hat{\vec{f}}_{m}\right\rangle_{2, m}
$$


So we clearly have that

$$
\hat{\overrightarrow{f_{m}}}=\underset{\vec{t}_{m} \in \mathcal{S}_{d_{1}}^{m}}{\operatorname{argmin}} \gamma_{n}\left(\vec{t}_{m}\right) .
$$

We define the penalty as

$$
\operatorname{pen}_{1}(m)=\frac{2 \kappa_{1} m}{n} \varrho^{2}\left(\mathbf{G}_{m}^{-1}\right)
$$

where $\kappa_{1}$ is a numerical constant see our comment below.

Theorem 4.2. Let us suppose that $(\boldsymbol{A} 1)-(\boldsymbol{A} 3)$ are true. Let $\hat{f}_{\widehat{m}_{1}}$ be defined by (9) and

$$
\widehat{m}_{1}=\underset{m \in \mathcal{M}_{n}^{(1)}}{\operatorname{argmin}}\left\{\gamma_{n}\left(\widehat{\vec{f}}_{m}\right)+\operatorname{pen}_{1}(m)\right\}
$$

with pen $_{1}$ defined by (20), then there exists a positive numerical constant $\kappa_{1}$ such that

$$
\mathbb{E}\left\|f-\hat{f}_{\widehat{m}_{1}}\right\|^{2} \leq 4 \inf _{m \in \mathcal{M}_{n}^{(1)}}\left\{\left\|f-f_{m}\right\|^{2}+\operatorname{pen}_{1}(m)\right\}+\frac{C}{n},
$$

where $C$ depends on $\|f\|$ and $\|g\|$.

It follows from the proof that $\kappa_{1}=32$ would suit. But in practice, values obtained from the theory are generally too large and the constant is calibrated by simulations. Once chosen, it remains fixed for all simulation experiments.

The oracle inequality (21) establishes a non asymptotic oracle bound. It shows that the squared bias variance tradeoff is automatically made up to a multiplicative constant. We have shown in Section 3 that the rates of convergence in deconvolution problems are intricate and depend on the regularity types of the function $f$ under estimation and the noise density $g$. Bias and variance order in Equation (14) as shown in Section 3 yield an optimal value of $m$ in function of $n$ which $m$ depends on unknown quantities, and thus cannot be implemented. That is why Equation (21) is of high interest: rates of convergence are reached without requiring to be specified in the framework.

Remark 5. Note it is common in the literature to assume that the distributions belong to a certain semiparametric model which is not the case in this paper. In the deconvolution setting with a Fourier approach, papers as Comte et al. (2006) for instance, assume that the Fourier transform of the target and error densities have a particular decay behavior. Here we only put some conditions on the form of the spectral norm of the matrix $\mathbf{G}_{m}^{-1}$. Indeed if Assumption (A3) is released then an adaptive procedure can still be obtained with the associated penalty $\operatorname{pen}(m)=2 \kappa m \varrho^{2}\left(\mathbf{G}_{m}^{-1}\right) \log (n) / n$. We can notice that if $\varrho^{2}\left(\mathbf{G}_{m}^{-1}\right)=\mathrm{O}\left(m^{\alpha}\right)$ then for any $\alpha$, Assumption (A3) is true. It is satisfied for instance for Gamma distributions. Similarly if $\varrho^{2}\left(\mathbf{G}_{m}^{-1}\right)=\mathrm{O}\left(m^{\alpha} e^{\lambda m^{\beta}}\right)$ then it is enough that $\beta<1$ to ensure (A3).

To our knowledge this is a new result in the literature.

4.2. Adaptive survival function estimation. In this particular framework, we make the two following assumptions:

(B2) $\mathcal{M}_{n}^{(2)}=\left\{1 \leq m \leq d_{2}, \frac{\varrho^{2}\left(\mathbf{G}_{m}^{-1}\right) \log n}{n} \leq C\right\}$, where $d_{2}<n$ may depend on $n$ and $C>0$.

(B3) $0<\mathbb{E}\left[Z_{1}^{3}\right]<\infty$.

For any $\vec{t} \in \mathbb{R}^{d_{2}}$, we define the following contrast for the survival function estimation

and we also have

$$
\delta_{n}(\vec{t})=\|\vec{t}\|_{2, d_{2}}^{2}-2\left\langle\vec{t}, \mathbf{G}_{d_{2}}^{-1}\left(\widehat{\vec{S}}_{Z, d_{2}}-\vec{S}_{Y, d_{2}}\right)\right\rangle_{2, d_{2}}
$$

$$
\left\langle\vec{t}_{m}, \mathbf{G}_{d_{2}}^{-1}\left(\widehat{\vec{S}}_{Z, d_{2}}-\vec{S}_{Y, d_{2}}\right)\right\rangle_{2, d_{2}}=\left\langle\vec{t}_{m}, \mathbf{G}_{m}^{-1}\left(\widehat{\vec{S}}_{Z, m}-\vec{S}_{Y, m}\right)\right\rangle_{2, m}=\left\langle\vec{t}_{m}, \widehat{\vec{S}}_{X, m}\right\rangle_{2, m}
$$

which yields that

$$
\widehat{\vec{S}}_{X, m}=\underset{\vec{t}_{m} \in \mathcal{S}_{d_{2}}^{m}}{\operatorname{argmin}} \delta_{n}\left(\vec{t}_{m}\right)
$$

We define the penalty as

$$
\operatorname{pen}_{2}(m)=\frac{\kappa_{2} \mathbb{E}\left[Z_{1}\right]}{n} \varrho^{2}\left(\mathbf{G}_{m}^{-1}\right) \log n
$$


Theorem 4.3. Let us suppose that (B1)-(B3) are true. Let $\widehat{S}_{X, \widehat{m}_{2}}$ be defined by (13) and

$$
\widehat{m}_{2}=\underset{m \in \mathcal{M}_{n}^{(2)}}{\operatorname{argmin}}\left\{\delta_{n}\left(\widehat{\vec{S}}_{X, m}\right)+\operatorname{pen}_{2}(m)\right\}
$$

with pen $_{2}$ defined by (22), then there exists a positive numerical constant $\kappa_{2}$ such that

$$
\mathbb{E}\left\|S_{X}-\widehat{S}_{X, \widehat{m}_{2}}\right\|^{2} \leq 4 \inf _{m \in \mathcal{M}_{n}^{(2)}}\left\{\left\|S_{X}-S_{X, m}\right\|^{2}+\operatorname{pen}_{2}(m)\right\}+\frac{C}{n},
$$

where $C$ is a constant depending on $\mathbb{E}\left[Z_{1}^{3}\right]$.

We can also notice that in the penalty associated with this procedure a logarithmic term appears while it was not in the upper bound of Equation (16). Such logarithms often appears in adaptive procedures.

Comments after Theorem 4.2 still hold. This oracle inequality shows that the squared bias variance tradeoff is automatically made. Asymptotically, this ensures that the rates of convergence are reached up to a $\log n$ factor. To our knowledge this the first time that a global adaptive procedure of the survival function is considered. This result rests upon the particularity of the Laguerre basis which enables to extend the adaptive estimation of the density function to the survival function.

Nevertheless this estimation cannot be computed directly since the penalty depends on the expectation of $Z$. A solution is to prove an oracle inequality for a random penalty associated to (22) which is made in the next corollary.

Corollary 4.4. Let us suppose that (B1)-(B3) are true. Let $\widehat{S}_{X, \tilde{m}_{2}}$ be defined by (13) and

$$
\begin{gathered}
\widetilde{m}_{2}=\underset{m \in \mathcal{M}_{n}^{(2)}}{\operatorname{argmin}}\left\{\delta_{n}\left(\widehat{\vec{S}}_{X, m}\right)+\widehat{\operatorname{pen}}_{2}(m)\right\} \\
\widehat{\operatorname{pen}}_{2}(m)=\frac{2 \kappa_{2} \bar{Z}_{n}}{n} \varrho^{2}\left(\mathbf{G}_{m}^{-1}\right) \log n \quad \text { where } \quad \bar{Z}_{n}=\frac{1}{n} \sum_{i=1}^{n} Z_{i},
\end{gathered}
$$

then there exists a positive numerical constant $\kappa_{2}$ such that

$$
\mathbb{E}\left\|S_{X}-\widehat{S}_{X, \widetilde{m}_{2}}\right\|^{2} \leq 4 \inf _{m \in \mathcal{M}_{n}^{(2)}}\left\{\left\|S_{X}-S_{X, m}\right\|^{2}+\operatorname{pen}_{2}(m)\right\}+\frac{C}{n}
$$

where $C$ is a constant depending on $\mathbb{E}\left[Z_{1}\right], \mathbb{E}\left[Z_{1}^{3}\right]$ and $\operatorname{Var}\left[Z_{1}\right]$.

\section{Illustrations}

The whole implementation is conducted using $\mathrm{R}$ software. The integrated squared errors $\left\|f-\hat{f}_{\widehat{m}}\right\|^{2}$ and $\left\|S_{X}-\widehat{S}_{X, \widetilde{m}_{2}}\right\|^{2}$ are computed via a standard approximation and discretization (over 300 points) of the integral on an interval of $\mathbb{R}$ respectively denoted by $I_{d}$ and $I_{S}$. Then the mean integrated squared errors (MISE) $\mathbb{E}\left\|f-\hat{f}_{\widehat{m}_{1}}\right\|^{2}$ and $\mathbb{E}\left\|S_{X}-\widehat{S}_{X, \tilde{m}_{2}}\right\|^{2}$ are computed as the empirical mean of the approximated ISE over 100 simulation samples.

The performance of the procedure is studied for the six following distributions for $Y$. Moreover all the densities are normalized with unit variance.

$\triangleright$ Exponential $\mathcal{E}(1), I_{d}=[0,5], I_{S}=[0,10]$.

$\triangleright$ Gamma distribution : $2 \cdot \Gamma\left(4, \frac{1}{4}\right), I_{d}=[0,10], I_{S}=[0,5]$.

$\triangleright$ Rayleigh distribution with $\sigma^{2}=2 /(4-\pi), f(x)=\frac{x}{\sigma^{2}} e^{-\frac{x^{2}}{2 \sigma^{2}}}, I_{d}=[0,5], I_{S}=[0,25]$.

$\triangleright$ Weibull, $X / \sqrt{\Gamma(4)-\Gamma(1+2 / 3)^{2}}, f(x)=\frac{k}{\lambda}\left(\frac{x}{\lambda}\right)^{k-1} e^{-(x / \lambda)^{k}} \mathbb{1}_{x \geq 0}$, with $k=\frac{3}{2}$ and $\lambda=1, I_{d}=[0,5]$, $I_{S}=[0,5]$.

$\triangleright$ Mixed Gamma distribution : $X=W / \sqrt{5.48}$, with $W \sim 0.4 \Gamma(5,1)+0.6 \Gamma(13,1), I_{d}=[0,26]$, $I_{S}=[0,15]$.

$\triangleright$ Chi-squared distribution with 10 degrees of freedom, $\chi^{2}(10) / \sqrt{20}, I_{d}=[0,10], I_{S}=[0,10]$.

Exponential and Weibull distributions are often used in survival and failure analysis. The Gamma distribution is also often used in insurance modelization. The Rayleigh distribution arises in wind velocity analysis for instance.

In the simulation the variance $\sigma^{2}$ of the error distribution $g$ takes the values $0,1 / 10$ and $1 / 4$. The case where the variance equals zero corresponds to the forward problem. In that case for the survival function, 
we simply compute the empirical estimator $S_{n}(x)=n^{-1} \sum_{i=1}^{n} \mathbb{1}\left\{X_{i}>x\right\}$ which reaches the parametric rate of convergence. We also use it as a benchmark for the quality of the estimation in the inverse problem. We are not aware of any other specific global method of deconvolution on the nonnegative real line. Dattner et al. (2011) propose a pointwise method and it would be difficult to compare the risk with our results. We then choose a Gamma distribution for the error distribution which verifies (C1)-(C3) for $r=2$.

$\triangleright$ Gamma noise: $\Gamma\left(2, \frac{1}{\sqrt{20}}\right)$ and $\Gamma\left(2, \frac{1}{\sqrt{8}}\right)$.

Thus the first Gamma distribution has a variance 1/10 and the second 1/4. We refer to Examples 1 and 2 for the computation of the matrix $\mathbf{G}_{m}$.

The adaptive procedure is then implemented as follows:

$\triangleright$ For $m \in \mathcal{M}_{n}=\left\{m_{1}, \ldots, m_{n}\right\}$, compute $-\left\|\hat{f}_{m}\right\|^{2}+\widehat{\operatorname{pen}}(m)$.

$\triangleright$ Choose $\hat{m}$ such that $\hat{m}=\underset{m \in \mathcal{M}_{n}}{\operatorname{argmin}}\left\{-\left\|\hat{f}_{m}\right\|^{2}+\widehat{\operatorname{pen}}(m)\right\}$.

$\triangleright$ And compute $\hat{f}_{\hat{m}}(x)=\sum_{k=0}^{\hat{m}-1} \hat{b}_{k}(f) \varphi_{k}(x)$.

The procedure is given for the density estimation. For the survival case the three steps are the same with the right quantities associated to the problem and described in Section 4.2. Besides, the penalties are chosen according to Theorem 4.1, Theorem 4.2, and Corollary 4.4. The constant calibrations were done with intensive preliminary simulations. We take $\kappa=0.1, \kappa_{1}=0.03$ and $\kappa_{3}=0.065$. We consider the following model collection $\mathcal{M}_{n}^{(2)}=\{m, \quad 0 \leq m \leq 30\}$ for the density and survival function estimation.

In order to measure the performances of our procedure (density estimation) to pre-existent literature, we also computed the MISE obtained with a classic deconvolution approach. More precisely, we apply the procedure of Comte et al. (2006). It corresponds to a projection method with a $\mathbb{R}$-supported sinus cardinal basis. Besides this procedure is minimax optimal in the case of a known ordinary smooth error distribution. We therefore compute the following estimator and penalty. Let $g^{*}$ refer to the Fourier transform of $g$ defined as $g^{*}(x)=\int e^{i u x} g(u) \mathrm{d} u$. For a Gamma distribution of parameter $\alpha$ and $\beta$, its Fourier transform is $g^{*}(u)=(1-i u / \beta)^{\alpha}$. We compute

$$
\hat{f}_{F, m}(x)=\frac{1}{2 \pi} \int_{-\pi m}^{\pi m} e^{-i x u} \frac{n^{-1} \sum_{j=1}^{n} e^{i u Z_{j}}}{g^{*}(u)} \mathrm{d} u,
$$

and select $m$ by minimizing $-\left\|\hat{f}_{F, m}\right\|^{2}+\operatorname{pen}_{F}^{(i)}(m), i=1,2$ where

$$
\operatorname{pen}_{F}^{(1)}(m)=\frac{\kappa_{F}^{(1)}}{2 \pi n} \int_{-\pi m}^{\pi m} \frac{\mathrm{d} u}{\left|g^{*}(u)\right|^{2}} \quad \text { and } \quad \operatorname{pen}_{F}^{(2)}(m)=\frac{\kappa_{F}^{(2)} m}{n} .
$$

The model collection is $\{m / 10: \quad m \in \mathbb{N}, \quad 1 \leq m \leq 20\}$. With pen ${ }_{F}^{(1)}$ we consider the inverse problem and with $\operatorname{pen}_{F}^{(2)}$ the forward problem. After calibration we find $\kappa_{F}^{(1)}=41$ and $\kappa_{F}^{(2)}=5$. Besides this procedure has the advantage of being a fast practical algorithm.

Results. The results are given in Tables 1 and 2. For both tables, the values of the MISE are multiplied by 100 for each case and computed from 100 simulated data. In Table 1 the abbreviations Lag and Fou correspond respectively to the Laguerre method and Fourier method of Comte et al. (2006). First we see that the risk decreases when the sample size increases. Likewise the risk increases when the variance of the noise increases. In the forward problem, we see that the Laguerre deconvolution has better performances than the Fourier deconvolution. For instance, when $n=2000$ the MISE in the Fourier setting is almost systematically twice larger than the Laguerre for the Gamma, Rayleigh, mixed Gamma and Chi-squared distributions between the Laguerre and Fourier methods. For the Exponential density estimation, the ratio of the MISE of Fourier divided by Laguerre is of 1 to 3 and for the Weibull distribution 1 to 33 . In the inverse problem, we can make the same kind of remarks noticing that the ratio is worse for the Fourier deconvolution: 1 to 18 .

Thus the results point out the relevance of a specific method for nonnegative variables in a deconvolution problem.

In Table 2, the first two columns correspond to the estimation with the empirical estimator of the survival function if we observe directly the data. The estimation is very good which was expected since the estimator converges to the true function with rate $\sqrt{n}$. Yet for the estimation of the Exponential distribution we note that the penalization procedure always beats the empirical estimator of the forward problem. It is also the 


\begin{tabular}{lccccccc}
\hline \hline & & & & & & \\
& & & $\sigma^{2}=0$ & $\sigma^{2}=\frac{1}{10}$ & $\sigma^{2}=\frac{1}{4}$ \\
& & 200 & 2000 & 200 & 2000 & 200 & 2000 \\
\hline \hline Exponential & Lag & 0.874 & 0.118 & 0.833 & 0.090 & 0.699 & 0.109 \\
& Fou & 3.950 & 3.234 & 6.002 & 3.359 & 11.228 & 3.889 \\
\hline Gamma & Lag & 0.243 & 0.048 & 1.181 & 0.076 & 1.872 & 0.384 \\
& Fou & 0.585 & 0.076 & 2.027 & 0.250 & 8.497 & 0.860 \\
\hline Rayleigh & Lag & 0.287 & 0.044 & 0.844 & 0.068 & 1.008 & 0.083 \\
& Fou & 0.498 & 0.073 & 1.546 & 0.248 & 7.523 & 0.797 \\
\hline Weibull & Lag & 1.714 & 0.275 & 8.518 & 1.529 & 13.768 & 3.543 \\
& Fou & 7.004 & 6.611 & 8.751 & 6.839 & 14.237 & 7.421 \\
\hline Mixed Gamma & Lag & 0.333 & 0.032 & 1.568 & 0.359 & 2.806 & 0.365 \\
& Fou & 0.488 & 0.062 & 1.038 & 0.204 & 8.317 & 0.829 \\
\hline Chi-squared & Lag & 0.357 & 0.037 & 0.443 & 0.260 & 2.861 & 0.315 \\
& Fou & 0.542 & 0.069 & 1.887 & 0.250 & 8.135 & 0.844 \\
\hline
\end{tabular}

TABLE 1. Results of simulation: MISE $\mathbb{E}\left(\left\|f-\hat{f}_{\widehat{m}}\right\|^{2}\right) \times 100$ averaged over 100 samples. The noise is $\Gamma\left(2, \frac{1}{\sqrt{20}}\right)$ for $\sigma^{2}=\frac{1}{10}$ and $\Gamma\left(2, \frac{1}{\sqrt{8}}\right)$ for $\sigma^{2}=\frac{1}{4}$.

\begin{tabular}{lcccccc}
\hline \hline & \multicolumn{2}{c}{$\sigma^{2}=0$} & \multicolumn{2}{c}{$\sigma^{2}=\frac{1}{10}$} & \multicolumn{2}{c}{$\sigma^{2}=\frac{1}{4}$} \\
& 200 & 2000 & 200 & 2000 & 200 & 2000 \\
\hline Exponential & 0.262 & 0.022 & 0.122 & 0.014 & 0.134 & 0.012 \\
Gamma & 0.263 & 0.024 & 0.688 & 0.203 & 1.363 & 0.233 \\
Rayleigh & 0.813 & 0.115 & 0.878 & 0.199 & 1.336 & 0.297 \\
Chi-squared & 0.310 & 0.027 & 1.313 & 0.117 & 1.445 & 0.679 \\
\hline
\end{tabular}

TABLE 2. Results of simulation: MISE $\mathbb{E}\left(\left\|S_{X}-\widehat{S}_{X, \tilde{m}_{2}}\right\|^{2}\right) \times 100$ averaged over 100 samples. The noise is $\Gamma\left(2, \frac{1}{\sqrt{20}}\right)$ for $\sigma^{2}=\frac{1}{10}$ and $\Gamma\left(2, \frac{1}{\sqrt{8}}\right)$ for $\sigma^{2}=\frac{1}{4}$.

case for the density estimation. It is explained by the fact that the Exponential density with parameter 1 corresponds to the first function of the basis. We notice that the risk decreases when the sample size increases. For the Exponential distribution, it is split by 10, by 3.5 for the Gamma distribution, by 4.5 for the Rayleigh distribution, by 13 for the Chi-squared distribution. And risk increases when the variance of the noise increases.

We also illustrate the results with some figures. Figures 1(a) and 1(b) show the estimated density and survival functions of the mixed Gamma in model selection along with beams of estimator.

\section{Concluding REMARKS}

This paper deals with the estimation of nonnegative variables in a deconvolution setting with a known error distribution. First we have considered the adaptive estimation of the density $f$ of the $X_{i}$ 's in a deconvolution setting and deduced a procedure when there is no additional noise. Secondly we have tackled the problem of the adaptive estimation of the survival function which is new to our knowledge, in a global estimation setting on $\mathbb{R}^{+}$. Moreover we have illustrated the performances of our new procedure and compared it, when it is possible to the performances of the Fourier procedure described in Comte et al. (2006). Our procedure outperforms the previous one. These results show that the Laguerre procedure is worthy of interest when the variables are nonnegative.

The assumption of the knowledge of the error distribution is often not realistic in applications. Nevertheless this would require additional information on the error distribution. In the deconvolution literature with unknown error distribution it is assumed that we have access to a preliminary sample of the noise, see for instance Neumann (1997). Thanks to this preliminary observation we could estimate the coefficients of the matrix $\mathbf{G}_{m}$. Vareschi (2014), in a Laplace regression model, considers this problem and assumes that a 
pertubation of the coefficients of the matrix $\mathbf{G}_{m}$ are observed. In our model we would need to control the deviation of the spectral norm of $\widehat{\mathbf{G}}_{m}^{-1}$ around the true spectral norm of $\mathbf{G}_{m}^{-1}$.

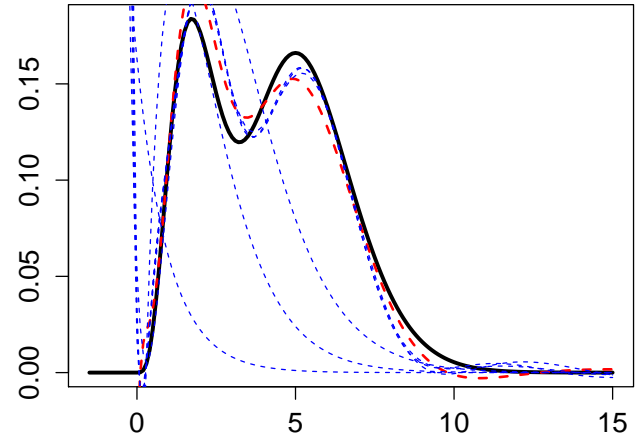

(a) Density estimation

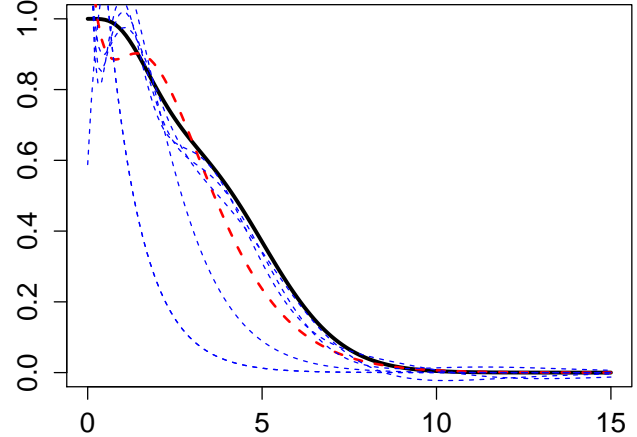

(b) Survival function estimation

FiguRE 1. Estimations for $n=500$ of a mixed Gamma (bold black line), the adaptive estimator (bold red line), beams of 5 estimators (blue dashed line).

\section{Proofs}

\subsection{Proof of Section 3.}

Proof of Proposition 3.1. Using Pythagora's Theorem, we have

$$
\left\|f-\hat{f}_{m}\right\|^{2}=\left\|f-f_{m}+f_{m}-\hat{f}_{m}\right\|^{2}=\left\|f-f_{m}\right\|^{2}+\left\|f_{m}-\hat{f}_{m}\right\|^{2} .
$$

The first term corresponds to the bias term of Equation (14). Let us study the second term: using the decomposition on the orthonormal Laguerre basis, we have

$$
\left\|f_{m}-\hat{f}_{m}\right\|^{2}=\sum_{k=0}^{m-1}\left(b_{k}(f)-\hat{b}_{k}(f)\right)^{2} .
$$

Finally we apply (6) and get

$$
\begin{aligned}
\mathbb{E}\left\|f_{m}-\hat{f}_{m}\right\|^{2} & =\mathbb{E}\left\|\mathbf{G}_{m}^{-1}\left(\hat{\vec{h}}_{m}-\vec{h}_{m}\right)\right\|_{2, m}^{2} \leq \varrho^{2}\left(\mathbf{G}_{m}^{-1}\right) \mathbb{E}\left\|\hat{\vec{h}}_{m}-\vec{h}_{m}\right\|_{2, m}^{2} \\
& \leq \varrho^{2}\left(\mathbf{G}_{m}^{-1}\right) \mathbb{E}\left[\sum_{j=1}^{m}\left(\frac{1}{n} \sum_{i=1}^{n} \varphi_{j}\left(Z_{i}\right)-\mathbb{E}\left[\varphi_{j}\left(Z_{1}\right)\right]\right)^{2}\right] \\
& \leq \frac{\varrho^{2}\left(\mathbf{G}_{m}^{-1}\right)}{n} \sum_{j=1}^{m} \mathbb{E}\left[\varphi_{j}^{2}\left(Z_{1}\right)\right] \leq \varrho^{2}\left(\mathbf{G}_{m}^{-1}\right) \frac{2 m}{n}
\end{aligned}
$$

In the end we get: $\mathbb{E}\left\|f-\hat{f}_{m}\right\|^{2} \leq\left\|f-f_{m}\right\|^{2}+\frac{2 m}{n} \varrho^{2}\left(\mathbf{G}_{m}^{-1}\right)$.

Proof of Proposition 3.3. As in the previous proof, we can write that

$$
\left\|S_{X}-\widehat{S}_{X, m}\right\|^{2}=\left\|S_{X}-S_{X, m}\right\|^{2}+\left\|S_{X, m}-\widehat{S}_{X, m}\right\|^{2} .
$$

We can notice that

$$
\left\|S_{X, m}-\widehat{S}_{X, m}\right\|^{2}=\left\|\widehat{\vec{S}}_{X, m}-\vec{S}_{X, m}\right\|_{2, m}^{2}=\left\|\mathbf{G}_{m}^{-1}\left(\widehat{\vec{S}}_{Z, m}-\vec{S}_{Z, m}\right)\right\|_{2, m}^{2}
$$


Then we repeat the same scheme as in the proof of Proposition 3.1 and we get

$$
\mathbb{E}\left\|S_{X, m}-\widehat{S}_{X, m}\right\|^{2} \leq \frac{1}{n} \varrho^{2}\left(\mathbf{G}_{m}^{-1}\right) \sum_{j=0}^{m-1} \mathbb{E}\left[\Phi_{j}^{2}\left(Z_{1}\right)\right]
$$

Yet

$$
\sum_{j=0}^{m-1} \Phi_{j}^{2}\left(Z_{1}\right)=\sum_{j=0}^{m-1}\left(\int \varphi_{j}(u) \mathbb{1}_{0 \leq u \leq Z_{1}} \mathrm{~d} u\right)^{2}=\sum_{j=0}^{m-1}\left\langle\varphi_{j}, \mathbb{1}_{. \leq Z_{1}}\right\rangle^{2} \leq\left\|\mathbb{1}_{. \leq Z_{1}}\right\|_{\mathbb{R}^{+}}^{2}=\int_{\mathbb{R}^{+}} \mathbb{1}_{u \leq Z_{1}} \mathrm{~d} u=Z_{1}
$$

which implies

$$
\mathbb{E}\left[\sum_{j=0}^{m-1} \Phi_{j}^{2}\left(Z_{1}\right)\right] \leq \mathbb{E}\left[Z_{1}\right]
$$

In the end: $\mathbb{E}\left\|S_{X}-\widehat{S}_{X, m}\right\|^{2} \leq\left\|S_{X}-S_{X, m}\right\|^{2}+\frac{\mathbb{E}\left[Z_{1}\right]}{n} \varrho^{2}\left(\mathbf{G}_{m}^{-1}\right)$.

Proof of Lemma 3.4. To see that the spectral norm grows with the dimension $m$, recall that for a matrix A of dimension $m$ the spectral norm can be written as $\varrho^{2}(\mathbf{A})=\max _{\|\vec{u}\|_{2, m}=1}\|\mathbf{A} \vec{u}\|_{2, m}^{2}$. Now consider $\vec{u}_{m}=\arg \max _{\|\vec{u}\|_{2, m}^{2}=1}\left\|\mathbf{T}_{m} \vec{u}\right\|_{2, m}$ with $\mathbf{T}_{m}$ a lower triangular matrix and $\mathbf{T}_{m}$ a submatrix of $\mathbf{T}_{m+1}$. We put $\vec{v}_{m+1}$ with $m$ first coordinates equal to $\vec{u}_{m}$. It yields that if we note $\left(a_{i}\right)_{1 \leq i \leq m+1}$ the coefficients of the vector $\mathbf{T}_{m+1} v_{m+1}$ we get

$$
\left\|\mathbf{T}_{m+1} \vec{v}_{m+1}\right\|_{2, m+1}^{2}=\sum_{i=1}^{m+1} a_{i}^{2}=\sum_{i=1}^{m} a_{i}^{2}+a_{m+1}^{2}=\left\|\mathbf{T}_{m} \vec{u}_{m}\right\|^{2}+a_{m+1}^{2} \geq \varrho^{2}\left(\mathbf{T}_{m}\right) .
$$

\subsection{Proof of Theorem 4.1.}

Proof of Theorem 4.1. Let $t, s \in \mathbb{L}^{2}\left(\mathbb{R}^{+}\right)$, we have

$$
\theta_{n}(t)-\theta_{n}(s)=\|t-f\|^{2}-\|s-f\|^{2}-2 \xi_{n}(t-s)
$$

with $\xi_{n}(t)=\frac{1}{n} \sum_{i=1}^{n}\left(t\left(X_{i}\right)-\langle t, f\rangle\right)$.

According to the definition of $\hat{m}$, for any $m$ in the model collection $\{1, \ldots, n\}$, we have the following inequality

It yields that

$$
\theta_{n}\left(\hat{f}_{\hat{m}}\right)+\operatorname{pen}(\hat{m}) \leq \theta_{n}\left(f_{m}\right)+\operatorname{pen}(m)
$$

which implies

$$
\left\|\hat{f}_{\hat{m}}-f\right\|^{2}-\left\|f_{m}-f\right\|^{2}-2 \xi_{n}\left(\hat{f}_{\hat{m}}-f_{m}\right) \leq \operatorname{pen}(m)-\operatorname{pen}(\hat{m})
$$

$\left\|\hat{f}_{\hat{m}}-f\right\|^{2} \leq\left\|f_{m}-f\right\|^{2}+2 \xi_{n}\left(\hat{f}_{\hat{m}}-f_{m}\right)+\operatorname{pen}(m)-\operatorname{pen}(\hat{m})$.

Since $t \mapsto \xi_{n}(t)$ is linear, we have that $\xi_{n}\left(\hat{f}_{\hat{m}}-f_{m}\right)=\left\|\hat{f}_{\hat{m}}-f_{m}\right\| \xi_{n}\left(\frac{\hat{f}_{\hat{m}}-f_{m}}{\left\|\hat{f}_{\hat{m}}-f_{m}\right\|}\right)$ and due to the relation $2 x y \leq x^{2} / 4+4 y^{2}$ we have the following inequalities

$$
\begin{aligned}
\left\|\hat{f}_{\hat{m}}-f\right\|^{2} & \leq\left\|f_{m}-f\right\|^{2}+2\left\|\hat{f}_{\hat{m}}-f_{m}\right\| \sup _{t \in B(m, \hat{m})} \xi_{n}(t)+\operatorname{pen}(m)-\operatorname{pen}(\hat{m}) \\
& \leq\left\|f_{m}-f\right\|^{2}+\frac{1}{4}\left\|\hat{f}_{\hat{m}}-f_{m}\right\|^{2}+4 \sup _{t \in B(m, \hat{m})} \xi_{n}^{2}(t)+\operatorname{pen}(m)-\operatorname{pen}(\hat{m})
\end{aligned}
$$

where $B(m, \hat{m})=\left\{t \in S_{m \vee \hat{m}},\|t\|=1\right\}$. Now let us notice that

$$
\left\|\hat{f}_{\hat{m}}-f_{m}\right\|^{2} \leq 2\left\|\hat{f}_{\hat{m}}-f\right\|^{2}+2\left\|f_{m}-f\right\|^{2} .
$$

Combining this latest inequality with Equation (26), we get

$$
\begin{aligned}
\left\|\hat{f}_{\hat{m}}-f\right\|^{2} & \leq\left\|f_{m}-f\right\|^{2}+\frac{1}{2}\left\|\hat{f}_{\hat{m}}-f\right\|^{2}+\frac{1}{2}\left\|f-f_{m}\right\|^{2}+4 \sup _{\vec{t} \in B(m, \hat{m})} \xi_{n}^{2}(\vec{t})+\operatorname{pen}(m)-\operatorname{pen}(\hat{m}) \\
& \leq 3\left\|f-f_{m}\right\|^{2}+2 \operatorname{pen}(m)+8 \sup _{t \in B(m, \hat{m})} \xi_{n}^{2}(\vec{t})-2 \operatorname{pen}(\hat{m})
\end{aligned}
$$


Now let $p$ be a function such that for any $m, m^{\prime}$, we have : $4 p\left(m, m^{\prime}\right) \leq \operatorname{pen}(m)+\operatorname{pen}\left(m^{\prime}\right)$.

$$
\begin{aligned}
\left\|\hat{f}_{\hat{m}}-f\right\|^{2} & \leq 3\left\|f-f_{m}\right\|^{2}+4 \operatorname{pen}(m)+8\left[\sup _{t \in B(m, \hat{m})} \xi_{n}^{2}(t)-p(m, \hat{m})\right]_{+} \\
& \leq 3\left\|f-f_{m}\right\|^{2}+4 \operatorname{pen}(m)+8 \sum_{m^{\prime} \in\{1, \ldots, n\}}\left\{\sup _{\vec{t} \in B\left(m, m^{\prime}\right)} \xi_{n}^{2}(t)-p\left(m, m^{\prime}\right)\right\}_{+}
\end{aligned}
$$

which implies that

$$
\mathbb{E}\left\|\hat{f}_{\hat{m}}-f\right\|^{2} \leq 4 \inf _{m \in\{1, \ldots, n\}}\left\{\left\|f-f_{m}\right\|^{2}+\operatorname{pen}(m)\right\}+8 \sum_{m^{\prime} \in\{1, \ldots, n\}} \mathbb{E}\left[\left\{\sup _{t \in B\left(m, m^{\prime}\right)} \xi_{n}^{2}(t)-p\left(m, m^{\prime}\right)\right\}\right] .
$$

We now use the following result which ensures the validity of Theorem 4.1.

Proposition 7.1. Under the assumptions of Theorem 4.1, there exists a constant $C>0$ depending on $\|f\|$ such that for $p\left(m, m^{\prime}\right)=\frac{2\left(m \vee m^{\prime}\right)}{n}$

$$
\sum_{m^{\prime} \in\{1, \ldots, n\}} \mathbb{E}\left[\left\{\sup _{t \in B\left(m, m^{\prime}\right)} \xi_{n}^{2}(t)-p\left(m, m^{\prime}\right)\right\}_{+}\right] \leq \frac{C}{n}
$$

Finally, $\mathbb{E}\left\|\hat{f}_{\hat{m}}-f\right\|^{2} \leq 4 \inf _{m \in\{1, \ldots, n\}}\left\{\left\|f-f_{m}\right\|^{2}+\operatorname{pen}(m)\right\}+\frac{C}{n}$.

Proof of Proposition 7.1. Let set $m^{*}=m \vee m^{\prime}$. Since $t \in \mathcal{S}_{m^{*}}, t(x)=\sum_{k=0}^{m^{*}-1} b_{k}(t) \varphi_{k}(x)$ and since $t \mapsto \xi_{n}(t)$ is linear we have

$$
\xi_{n}(t)=\sum_{k=0}^{m^{*}-1} b_{k}(t) \xi_{n}\left(\varphi_{k}\right)
$$

Now, applying Cauchy-Schwarz inequality we get, using $\|t\|=1$

$$
\left(\xi_{n}(t)\right)^{2} \leq \sum_{k=0}^{m^{*}-1} b_{k}^{2}(t) \sum_{k=0}^{m^{*}-1} \xi_{n}^{2}\left(\varphi_{k}\right)=\|t\|^{2} \sum_{k=0}^{m^{*}-1} \xi_{n}^{2}\left(\varphi_{k}\right)=\sum_{k=0}^{m^{*}-1} \xi_{n}^{2}\left(\varphi_{k}\right)
$$

which implies

$$
\mathbb{E}\left[\sup _{t \in B\left(m, m^{\prime}\right)}\left(\xi_{n}(t)\right)^{2}\right] \leq \sum_{k=0}^{m^{*}-1} \mathbb{E}\left[\xi_{n}^{2}\left(\varphi_{k}\right)\right] .
$$

To apply Talagrand inequality we compute $H^{2}, M_{1}$ and $v$ as defined in Appendix A. The empirical process can be bounded as follows

$$
\mathbb{E}\left[\sup _{t \in B\left(m, m^{\prime}\right)}\left(\xi_{n}(t)\right)^{2}\right] \leq \sum_{k=0}^{m^{*}-1} \mathbb{E}\left[\frac{1}{n^{2}} \sum_{i=1}^{n}\left(\varphi_{k}\left(X_{i}\right)-\left\langle\varphi_{k}, f\right\rangle\right)^{2}\right] \leq \frac{1}{n} \sum_{k=0}^{m^{*}-1} \mathbb{E}\left[\varphi_{k}^{2}\left(X_{1}\right)\right] \leq \frac{2 m^{*}}{n}:=H^{2} .
$$

Moreover applying Cauchy-Schwarz inequality

$$
\operatorname{Var}\left[t\left(X_{1}\right)\right] \leq \mathbb{E}\left[t\left(X_{1}\right)^{2}\right] \leq \int_{\mathbb{R}^{+}} t^{2}(x) f(x) \mathrm{d} x \leq\|t\|_{\infty}\|t\|\|f\| \leq \sqrt{2 m^{*}}\|f\|:=\nu
$$

and

$$
\|t\|_{\infty} \leq \sqrt{2 m^{*}}\|t\|=\sqrt{2 m^{*}}:=M_{1} .
$$

So applying Talagrand's inequality we get

$$
\mathbb{E}\left[\left\{\sup _{t \in B_{m, m^{\prime}}}\left|\xi_{n}(t)\right|^{2}-8 \frac{m^{*}}{n}\right\}_{+}\right] \leq \frac{6}{K_{1}}\left(\frac{\sqrt{2 m^{*}}\|f\|}{n} e^{-C_{1} \sqrt{m^{*}} /\|f\|}+\frac{16 n}{C_{2} n^{2}} e^{-C_{3} \sqrt{n}}\right)
$$

which implies

$$
\sum_{m^{\prime} \in\{1, \ldots, n\}} \mathbb{E}\left[\left\{\sup _{t \in B_{m, m^{\prime}}}\left|\xi_{n}(t)\right|^{2}-8 \frac{m^{*}}{n}\right\}_{+}\right] \leq \sum_{m^{\prime} \in\{1, \ldots, n\}} \frac{6}{K_{1}}\left(\frac{\sqrt{2 m^{*}}\|f\|}{n} e^{-C_{1} m^{*} /\|f\|}+\frac{16}{C_{2} n} e^{-C_{3} \sqrt{n}}\right) \leq \frac{C}{n} .
$$




\subsection{Proof of Theorem 4.2.}

Proof of Theorem 4.2. Let $m, m^{\prime} \in \mathcal{M}_{n}^{(1)}, \vec{t}_{m} \in \mathcal{S}_{d_{1}}^{m}$ and $\vec{s}_{m^{\prime}} \in \mathcal{S}_{d_{1}}^{m^{\prime}}$. Denote $m^{*}=m \vee m^{\prime}$. Notice that

$$
\gamma_{n}\left(\vec{t}_{m}\right)-\gamma_{n}\left(\vec{s}_{m^{\prime}}\right)=\left\|\vec{t}_{m}-\vec{f}\right\|_{2, d_{1}}^{2}-\left\|\vec{s}_{m^{\prime}}-\vec{f}\right\|_{2, d_{1}}^{2}-2\left\langle\vec{t}_{m}-\vec{s}_{m^{\prime}}, \mathbf{G}_{d_{1}}^{-1}\left(\hat{\vec{h}}_{d_{1}}-\vec{h}_{d_{1}}\right)\right\rangle_{2, d_{1}}
$$

and due to orthonormality of Laguerre basis, for any $m$ we have the following relations between the $\mathbb{L}^{2}$ norm and the Euclidean norms,

$$
\left\|\hat{f}_{m}-f\right\|^{2}=\left\|\hat{\vec{f}}_{m}-\vec{f}\right\|_{2, d_{1}}^{2}+\sum_{j=d_{1}}^{\infty}\left(b_{j}(f)\right)^{2} \quad \text { and } \quad\left\|f_{m}-f\right\|^{2}=\left\|\vec{f}_{m}-\vec{f}\right\|_{2, d_{1}}^{2}+\sum_{j=d_{1}}^{\infty}\left(b_{j}(f)\right)^{2}
$$

We set $\nu_{n}(\vec{t})=\left\langle\vec{t}, \mathbf{G}_{d_{1}}^{-1}\left(\hat{\vec{h}}_{d_{1}}-\vec{h}_{d_{1}}\right)\right\rangle_{2, d_{1}}$ for $\vec{t} \in \mathbb{R}^{d_{1}}$.

According to the definition of $\widehat{m}$, for any $m$ in the model collection $\mathcal{M}_{n}^{(1)}$, we have the following inequality

$$
\gamma_{n}\left(\hat{\vec{f}}_{\widehat{m}}\right)+\operatorname{pen}_{1}(\widehat{m}) \leq \gamma_{n}\left(\vec{f}_{m}\right)+\operatorname{pen}_{1}(m) .
$$

It yields that

$$
\left\|\hat{\vec{f}}_{\widehat{m}}-\vec{f}\right\|_{2, d_{1}}^{2}-\left\|\vec{f}_{m}-\vec{f}\right\|_{2, d_{1}}^{2}-2 \nu_{n}\left(\hat{\vec{f}}_{\widehat{m}}-\vec{f}_{m}\right) \leq \operatorname{pen}_{1}(m)-\operatorname{pen}_{1}(\widehat{m})
$$

which implies

$$
\left\|\hat{\vec{f}}_{\widehat{m}}-\vec{f}\right\|_{2, d_{1}}^{2} \leq\left\|\vec{f}_{m}-\vec{f}\right\|_{2, d_{1}}^{2}+2 \nu_{n}\left(\hat{\vec{f}}_{\widehat{m}}-\vec{f}_{m}\right)+\operatorname{pen}_{1}(m)-\operatorname{pen}_{1}(\widehat{m})
$$

Let us notice that $\nu_{n}\left(\hat{\vec{f}}_{\widehat{m}}-\vec{f}_{m}\right)=\left\|\hat{\vec{f}}_{\widehat{m}}-\vec{f}_{m}\right\|_{2, d_{1}} \nu_{n}\left(\frac{\hat{\overrightarrow{f_{\hat{m}}}}-\vec{f}_{m}}{\left\|\hat{\vec{f}}_{\widehat{m}}-\vec{f}_{m}\right\|_{2, d_{1}}}\right)$ and due to the relation $2 a b \leq$ $a^{2} / 4+4 b^{2}$, we have the following inequalities

$$
\begin{aligned}
\left\|\hat{\vec{f}}_{\widehat{m}}-\vec{f}\right\|_{2, d_{1}}^{2} & \leq\left\|\vec{f}_{m}-\vec{f}\right\|_{2, d_{1}}^{2}+2\left\|\hat{\vec{f}}_{\widehat{m}}-\vec{f}_{m}\right\|_{2, d_{1}} \sup _{\vec{t} \in \mathcal{B}(m, \widehat{m})} \nu_{n}(\vec{t})+\operatorname{pen}_{1}(m)-\operatorname{pen}_{1}(\widehat{m}) \\
& \leq\left\|\vec{f}_{m}-\vec{f}\right\|_{2, d_{1}}^{2}+\frac{1}{4}\left\|\hat{\vec{f}}_{\widehat{m}}-\vec{f}_{m}\right\|_{2, d_{1}}^{2}+4 \sup _{\vec{t} \in \mathcal{B}(m, \widehat{m})} \nu_{n}^{2}(\vec{t})+\operatorname{pen}_{1}(m)-\operatorname{pen}_{1}(\widehat{m})
\end{aligned}
$$

where $\mathcal{B}(m, \widehat{m})=\left\{\vec{t}_{m \vee \widehat{m}} \in \mathcal{S}_{d_{1}}^{m \vee \widehat{m}},\left\|\vec{t}_{m \vee \widehat{m}}\right\|_{2, d_{1}}=1\right\}$. Now notice that

$$
\left\|\hat{\vec{f}}_{\widehat{m}}-\vec{f}_{m}\right\|_{2, d_{1}}^{2} \leq 2\left\|\hat{\vec{f}}_{\widehat{m}}-\vec{f}\right\|_{2, d_{1}}^{2}+2\left\|\vec{f}_{m}-\vec{f}\right\|_{2, d_{1}}^{2}
$$

we then have

$$
\left\|\hat{\vec{f}}_{\widehat{m}}-\vec{f}\right\|_{2, d_{1}}^{2} \leq\left\|\vec{f}_{m}-\vec{f}\right\|_{2, d_{1}}^{2}+\frac{1}{2}\left\|\hat{\vec{f}}_{\widehat{m}}-\vec{f}\right\|_{2, d_{1}}^{2}+\frac{1}{2}\left\|\vec{f}-\vec{f}_{m}\right\|_{2, d_{1}}^{2}+4 \sup _{\vec{t} \in \mathcal{B}(m, \widehat{m})} \nu_{n}^{2}(\vec{t})+\operatorname{pen}_{1}(m)-\operatorname{pen}_{1}(\widehat{m})
$$

which implies

$$
\left\|\hat{\vec{f}}_{\widehat{m}}-\vec{f}\right\|_{2, d_{1}}^{2} \leq 3\left\|\vec{f}-\vec{f}_{m}\right\|_{2, d_{1}}^{2}+2 \operatorname{pen}_{1}(m)+8 \sup _{\vec{t} \in \mathcal{B}(m, \widehat{m})} \nu_{n}^{2}(\vec{t})-2 \operatorname{pen}_{1}(\widehat{m}) .
$$

Using Equation (27), we have

$$
\left\|\hat{f}_{\widehat{m}}-f\right\|^{2}-\sum_{j=d_{1}}^{\infty}\left(b_{j}(f)\right)^{2} \leq 3\left(\left\|f-f_{m}\right\|^{2}-\sum_{j=d_{1}}^{\infty}\left(b_{j}(f)\right)^{2}\right)+2 \operatorname{pen}_{1}(m)+8 \sup _{\vec{t} \in \mathcal{B}(m, \widehat{m})} \nu_{n}^{2}(\vec{t})-2 \operatorname{pen}_{1}(\widehat{m})
$$

which implies

$$
\left\|\hat{f}_{\widehat{m}}-f\right\|^{2} \leq 3\left\|f-f_{m}\right\|^{2}+2 \operatorname{pen}_{1}(m)+8 \sup _{\vec{t} \in \mathcal{B}(m, \widehat{m})} \nu_{n}^{2}(\vec{t})-2 \operatorname{pen}_{1}(\widehat{m})
$$

Now let $p_{1}$ be a function such that for any $m, m^{\prime}$, we have : $4 p_{1}\left(m, m^{\prime}\right) \leq \operatorname{pen}_{1}(m)+\operatorname{pen}_{1}\left(m^{\prime}\right)$.

$$
\begin{aligned}
\left\|\hat{f}_{\widehat{m}}-f\right\|^{2} & \leq 3\left\|f-f_{m}\right\|^{2}+4 \operatorname{pen}_{1}(m)+8\left[\sup _{\vec{t} \in \mathcal{B}(m, \widehat{m})} \nu_{n}^{2}(\vec{t})-p_{1}(m, \widehat{m})\right]_{+} \\
& \leq 3\left\|f-f_{m}\right\|^{2}+4 \operatorname{pen}_{1}(m)+8 \sum_{m^{\prime} \in \mathcal{M}_{n}^{(1)}}\left\{\sup _{\vec{t} \in \mathcal{B}\left(m, m^{\prime}\right)} \nu_{n}^{2}(\vec{t})-p_{1}\left(m, m^{\prime}\right)\right\}_{+}
\end{aligned}
$$

We now use the following result which ensures the validity of Theorem 4.2. 
Proposition 7.2. Under the assumptions of Theorem 4.2, then there exists a constant $C_{1}>0$ depending on $\|h\|_{\infty}$ such that for $p_{1}\left(m, m^{\prime}\right)=\frac{8\left(m \vee m^{\prime}\right)}{n} \varrho^{2}\left(\mathbf{G}_{m \vee m^{\prime}}^{-1}\right)$

$$
\mathbb{E}\left[\left\{\sup _{\vec{t} \in \mathcal{B}\left(m, m^{\prime}\right)} \nu_{n}^{2}(\vec{t})-p\left(m, m^{\prime}\right)\right\}_{+}\right] \leq \frac{C_{1}}{n} .
$$

In the end: $\mathbb{E}\left\|f-\hat{f}_{\widehat{m}}\right\|^{2} \leq 4 \inf _{m \in \mathcal{M}_{n}^{(1)}}\left\{\left\|f-f_{m}\right\|^{2}+\operatorname{pen}_{1}(m)\right\}+\frac{C_{1}}{n}$, as soon as $\kappa_{1} \geq 32$.

Proof of Proposition 7.2. To prove Proposition 7.2, we apply a Talagrand inequality. So we need to determine $H, M_{1}$ and $v$ defined as

$$
\begin{aligned}
& \sup _{\vec{t}_{m^{*}} \in \mathcal{B}\left(m, m^{\prime}\right)}\left\|\left\langle\vec{t}_{m^{*}}, \mathbf{G}_{d_{1}}^{-1} \vec{\varphi}_{d_{1}}(.)\right\rangle_{2, d_{1}}\right\|_{\infty} \leq M_{1}, \quad \mathbb{E}\left[\sup _{\vec{t}_{m^{*}} \in \mathcal{B}\left(m, m^{\prime}\right)}\left|\nu_{n}\left(\vec{t}_{m^{*}}\right)\right|\right] \leq H, \\
& \sup _{\vec{t}_{m^{*}} \in \mathcal{B}\left(m, m^{\prime}\right)} \operatorname{Var}\left[\left\langle\vec{t}_{m^{*}}, \mathbf{G}_{d_{1}}^{-1} \vec{\varphi}_{d_{1}}\left(Z_{1}\right)\right\rangle_{2, d_{1}}\right] \leq v .
\end{aligned}
$$

where $m^{*}=m \vee m^{\prime}$.

- Let us start with the empirical process, first let us notice that

$$
\begin{aligned}
\mathbb{E}\left[\sup _{\vec{t}_{m^{*}} \in \mathcal{B}\left(m, m^{\prime}\right)}\left|\nu_{n}\left(\vec{t}_{m^{*}}\right)\right|^{2}\right] & =\mathbb{E}\left[\sup _{\vec{t}_{m^{*}} \in \mathcal{B}\left(m, m^{\prime}\right)}\left|\left\langle\vec{t}_{m^{*}}, \mathbf{G}_{d_{1}}^{-1}\left(\frac{1}{n} \sum_{i=1}^{n}\left(\vec{\varphi}_{d_{1}}\left(Z_{i}\right)-\mathbb{E}\left[\vec{\varphi}_{d_{1}}\left(Z_{i}\right)\right]\right)\right)\right\rangle_{2, d_{1}}\right|^{2}\right] \\
& =\mathbb{E}\left[\sup _{\vec{t}_{m^{*}} \in \mathcal{B}\left(m, m^{\prime}\right)}\left|\left\langle\vec{t}_{m^{*}}, \mathbf{G}_{m^{*}}^{-1}\left(\frac{1}{n} \sum_{i=1}^{n}\left(\vec{\varphi}_{m^{*}}\left(Z_{i}\right)-\mathbb{E}\left[\vec{\varphi}_{m^{*}}\left(Z_{i}\right)\right]\right)\right)\right\rangle_{2, m^{*}}\right|^{2}\right]
\end{aligned}
$$

We now apply Cauchy-Schwarz inequality and get

$$
\begin{aligned}
& \mathbb{E}\left[\sup _{\vec{t}_{m^{*}} \in \mathcal{B}\left(m, m^{\prime}\right)}\left|\nu_{n}\left(\vec{t}_{m^{*}}\right)\right|^{2}\right] \\
& \leq \mathbb{E}\left[\sup _{\vec{t}_{m^{*}} \in \mathcal{B}\left(m, m^{\prime}\right)}\left\|\vec{t}_{m^{*}}\right\|_{2, m^{*}}^{2}\left\|\mathbf{G}_{m^{*}}^{-1}\left(\frac{1}{n} \sum_{i=1}^{n}\left(\vec{\varphi}_{m^{*}}\left(Z_{i}\right)-\mathbb{E}\left[\vec{\varphi}_{m^{*}}\left(Z_{i}\right)\right]\right)\right)\right\|_{2, m^{*}}^{2}\right] \\
& \leq \varrho^{2}\left(\mathbf{G}_{m^{*}}^{-1}\right) \mathbb{E}\left[\sum_{j=0}^{m^{*}-1}\left(\frac{1}{n} \sum_{i=1}^{n}\left(\varphi_{j}\left(Z_{i}\right)-\mathbb{E}\left[\varphi_{j}\left(Z_{i}\right)\right]\right)\right)^{2}\right] \\
& \leq \frac{\varrho^{2}\left(\mathbf{G}_{m^{*}}^{-1}\right)}{n} \sum_{j=0}^{m^{*}-1} \operatorname{Var}\left(\varphi_{j}\left(Z_{1}\right)\right) \leq \frac{\varrho^{2}\left(\mathbf{G}_{m^{*}}^{-1}\right)}{n} \sum_{j=0}^{m^{*}-1} \mathbb{E}\left[\varphi_{j}^{2}\left(Z_{1}\right)\right] \leq \frac{2 m^{*}}{n} \varrho^{2}\left(\mathbf{G}_{m^{*}}^{-1}\right) .
\end{aligned}
$$

We then set $H:=\sqrt{\frac{2 m^{*}}{n} \varrho^{2}\left(\mathbf{G}_{m^{*}}^{-1}\right)}$.

Before deriving a bound for the term of variance, let us remind that for any $x \in \mathbb{R}^{+}$, we have

$$
h(x)=\int f(u) g(x-u) \mathbb{1}_{u \geq 0} \mathbb{1}_{x-u \geq 0} \mathrm{~d} u=\int_{0}^{x} f(u) g(x-u) \mathrm{d} u .
$$

Then we apply Cauchy-Schwarz inequality and get $\forall x \in \mathbb{R}^{+}, h(x) \leq\|f\|\|g\|$. If Assumption (A1) is fulfilled, we clearly have $\|h\|_{\infty} \leq\|f\|\|g\|<\infty$.

- Now for the term of variance, let $\vec{t}_{m^{*}} \in \mathcal{B}\left(m, m^{\prime}\right)$. By definition we have the following equalities

$$
\begin{aligned}
\mathbb{E}\left[\left|\left\langle\vec{t}_{m^{*}}, \mathbf{G}_{d_{1}}^{-1} \vec{\varphi}_{d_{1}}\left(Z_{1}\right)\right\rangle_{2, d_{1}}\right|^{2}\right] & =\mathbb{E}\left[\left|\left\langle\vec{t}_{m^{*}}, \mathbf{G}_{m^{*}}^{-1} \vec{\varphi}_{m^{*}}\left(Z_{1}\right)\right\rangle_{2, m^{*}}\right|^{2}\right]=\mathbb{E}\left[\left|\sum_{j=0}^{m^{*}-1} b_{j}(t) \sum_{k=0}^{m^{*}-1} g_{j k} \varphi_{k}\left(Z_{1}\right)\right|^{2}\right] \\
& =\int_{\mathbb{R}^{+}}\left|\sum_{0 \leq k, j \leq m^{*}-1} b_{j}(t) g_{j k} \varphi_{k}(u)\right|^{2} h(u) \mathrm{d} u .
\end{aligned}
$$


which implies

$$
\begin{aligned}
\mathbb{E} & {\left[\left|\left\langle\vec{t}_{m^{*}}, \mathbf{G}_{d_{1}}^{-1} \vec{\varphi}_{d_{1}}\left(Z_{1}\right)\right\rangle_{2, d_{1}}\right|^{2}\right] } \\
& \leq\|h\|_{\infty} \int_{\mathbb{R}^{+}} \sum_{0 \leq j, j^{\prime}, k, k^{\prime} \leq m^{*}-1} b_{j}(t) b_{j^{\prime}}(t) g_{j k} g_{j^{\prime} k^{\prime}} \varphi_{k}(u) \varphi_{k^{\prime}}(u) \mathrm{d} u \\
& \leq\|h\|_{\infty} \sum_{0 \leq j, j^{\prime}, k, k^{\prime} \leq m^{*}-1} b_{j}(t) b_{j^{\prime}}(t) g_{j k} g_{j^{\prime} k^{\prime}} \delta_{k, k^{\prime}} \leq\|h\|_{\infty} \sum_{0 \leq j, j^{\prime}, k \leq m^{*}-1} b_{j}(t) b_{j^{\prime}}(t) g_{j k} g_{j^{\prime} k} \\
& \leq\|h\|_{\infty}{ }^{t} \vec{t}_{m^{*}} \mathbf{G}_{m^{*}}^{-1} \mathbf{G}_{m^{*}}^{-1} \vec{t}_{m^{*}} \leq\|h\|_{\infty} \varrho^{2}\left(\mathbf{G}_{m^{*}}^{-1}\right)\left\|\vec{t}_{m^{*}}\right\|_{2, m^{*}}^{2} \leq\|h\|_{\infty} \varrho^{2}\left(\mathbf{G}_{m^{*}}^{-1}\right) .
\end{aligned}
$$

So we set $v:=\|h\|_{\infty} \varrho^{2}\left(\mathbf{G}_{m^{*}}^{-1}\right)$.

- Now applying Cauchy-Schwarz inequality

$$
\begin{aligned}
& \quad \sup _{\vec{t}_{m^{*}} \in \mathcal{B}\left(m, m^{\prime}\right)} \sup _{x \in \mathbb{R}^{+}}\left|\left\langle\vec{t}_{m^{*}}, \mathbf{G}_{d_{1}}^{-1} \vec{\varphi}_{d_{1}}(x)\right\rangle_{2, d_{1}}\right|=\sup _{\vec{t}_{m^{*}} \in \mathcal{B}\left(m, m^{\prime}\right)} \sup _{x \in \mathbb{R}^{+}}\left|\left\langle\vec{t}_{m^{*}}, \mathbf{G}_{m^{*}}^{-1} \vec{\varphi}_{m^{*}}(x)\right\rangle_{2, m^{*}}\right| \\
& \leq \sup _{\vec{t}_{m^{*}} \in \mathcal{B}\left(m, m^{\prime}\right)} \sup _{x \in \mathbb{R}^{+}}\left\|\vec{t}_{m^{*}}\right\|_{2, m^{*}}\left\|\mathbf{G}_{m^{*}}^{-1} \vec{\varphi}_{m^{*}}(x)\right\|_{2, m^{*}} \leq \sup _{x \in \mathbb{R}^{+}}\left\|\mathbf{G}_{m^{*}}^{-1} \vec{\varphi}_{m^{*}}(x)\right\|_{2, m^{*}} \\
& \leq \sup _{x \in \mathbb{R}^{+}} \sqrt{t_{\vec{\varphi}_{m^{*}}}(x)^{t} \mathbf{G}_{m^{*}}^{-1} \mathbf{G}_{m^{*}}^{-1} \vec{\varphi}_{m^{*}}(x)} \leq \sqrt{\varrho^{2}\left(\mathbf{G}_{m^{*}}^{-1}\right) \sup _{x \in \mathbb{R}^{+}} \sum_{j=0}^{m^{*}-1} \varphi_{j}^{2}(x) \leq \sqrt{2 m^{*} \varrho^{2}\left(\mathbf{G}_{m^{*}}^{-1}\right)} .}
\end{aligned}
$$

We take $M_{1}=\sqrt{2 m^{*} \varrho^{2}\left(\mathbf{G}_{m^{*}}^{-1}\right)}$.

- We can now apply Talagrand's inequality for $\xi^{2}=1 / 2$

$\mathbb{E}\left[\left\{\sup _{\vec{t} \in \mathcal{B}\left(m, m^{\prime}\right)}\left|\nu_{n}(\vec{t})\right|^{2}-8 m^{*} \varrho^{2}\left(\mathbf{G}_{m^{*}}^{-1}\right)\right\}_{+}\right] \leq C_{1} \frac{\|h\|_{\infty} \varrho^{2}\left(\mathbf{G}_{m^{*}}^{-1}\right)}{n} e^{-C_{2} a m^{*} /\|h\|_{\infty}}+C_{3} \frac{m^{*} \varrho^{2}\left(\mathbf{G}_{m^{*}}^{-1}\right)}{n^{2}} e^{-C_{4} \sqrt{n}}$

which implies that

$$
\begin{aligned}
& \sum_{m^{\prime} \in \mathcal{M}_{n}^{(1)}} \mathbb{E}\left[\left\{\sup _{\vec{t} \in \mathcal{B}\left(m, m^{\prime}\right)}\left|\nu_{n}(\vec{t})\right|^{2}-8 m^{*} \varrho^{2}\left(\mathbf{G}_{m^{*}}^{-1}\right)\right\}_{+}\right] \\
& \leq C \sum_{m^{\prime} \in \mathcal{M}_{n}^{(1)}} \frac{\|h\|_{\infty} \varrho^{2}\left(\mathbf{G}_{m^{*}}^{-1}\right)}{n} e^{-C_{2} \frac{m^{*}}{\|h\|_{\infty}}+\frac{m^{*} \varrho^{2}\left(\mathbf{G}_{m^{*}}^{-1}\right)}{n^{2}} e^{-C_{4} \sqrt{n}}}
\end{aligned}
$$

Yet under Assumption (A3), we have

$$
\sum_{m^{\prime} \in \mathcal{M}_{n}^{(1)}} \frac{\|h\|_{\infty} \varrho^{2}\left(\mathbf{G}_{m^{*}}^{-1}\right)}{n} e^{-C_{2} \frac{m^{*}}{\|h\|_{\infty}}} \leq \frac{C}{n}
$$

Moreover according to Assumption (A2), we also have

$$
\sum_{m^{\prime} \in \mathcal{M}_{n}^{(1)}} \frac{m^{*} \varrho^{2}\left(\mathbf{G}_{m^{*}}^{-1}\right)}{n^{2}} e^{-C_{4} n} \leq \sum_{m^{\prime} \in \mathcal{M}_{n}^{(1)}} \frac{e^{-C_{4} \sqrt{n}}}{n} \leq \frac{C}{n}
$$

In the end we have the desired result.

Proof of Remark 5. We have to prove that Proposition 7.2 is still valid although Assumption (A3) is no longer true. We set $\xi^{2}=\frac{2 \log n}{K_{1}}, H^{2}=\frac{2 m^{*} \varrho^{2}\left(\mathbf{G}_{m^{*}}^{-1}\right)}{n}, v=2 m^{*} \varrho^{2}\left(\mathbf{G}_{m^{*}}^{-1}\right), M_{1}=\sqrt{2 m^{*} \varrho^{2}\left(\mathbf{G}_{m^{*}}^{-1}\right)}$. Under (A2), we have

$$
\sum_{m^{\prime} \in \mathcal{M}_{n}^{(1)}} \frac{v}{n} \exp \left(-K_{1} \xi^{2} n \frac{H^{2}}{v}\right)=\sum_{m^{\prime} \in \mathcal{M}_{n}^{(1)}} \frac{2 m^{*} \varrho^{2}\left(\mathbf{G}_{m^{*}}^{-1}\right)}{n} \frac{1}{n^{2}} \leq \sum_{m^{\prime} \in \mathcal{M}_{n}^{(1)}} \frac{2}{n^{2}} \leq \frac{2\left|\mathcal{M}_{n}^{(1)}\right|}{n^{2}} \leq \frac{2}{n} .
$$


For $C(\xi)$ defined in Lemma A.1, we get

$$
\begin{aligned}
& \sum_{m^{\prime} \in \mathcal{M}_{n}^{(1)}} \frac{M_{1}^{2}}{K_{1} C^{2}\left(\xi^{2}\right) n^{2}} \exp \left(-K_{1} \frac{C(\xi) \xi n H}{\sqrt{2} M_{1}}\right) \\
& =\sum_{m^{\prime} \in \mathcal{M}_{n}^{(1)}} \frac{m^{*} \varrho^{2}\left(\mathbf{G}_{m^{*}}^{-1}\right)}{K_{1} C^{2}\left(\xi^{2}\right) n^{2}} \exp \left(-K_{1} C(\xi) \xi \sqrt{n}\right) \leq \sum_{m^{\prime} \in \mathcal{M}_{n}^{(1)}} \frac{C_{2} m^{*} \varrho^{2}\left(\mathbf{G}_{m^{*}}^{-1}\right)}{(\log n)^{2} n^{2}} \exp \left(-C_{1} \log n \sqrt{n}\right) \\
& \leq \frac{C_{3}\left|\mathcal{M}_{n}^{(1)}\right|}{(\log n)^{2} n} \exp \left(-C_{1} \log n \sqrt{n}\right) \leq \frac{C_{3}}{n} .
\end{aligned}
$$

\subsection{Proof of Theorem 4.3 and Corollary 4.4.}

Proof of Theorem 4.3. The beginning of the proof is the same as the proof of Theorem 4.2 with the quantities associated to the survival function estimation. Then we start from Equation (28) with $\nu_{n}(\vec{t})$ replacing the following empirical process $\zeta_{n}(\vec{t}):=\zeta_{n}^{(1)}(\vec{t})+\zeta_{n}^{(2)}(\vec{t})$ where,

$$
\begin{aligned}
\zeta_{n}^{(1)}(\vec{t}) & :=\left\langle\vec{t}, \mathbf{G}_{d_{2}}^{-1}\left(\frac{1}{n} \sum_{i=1}^{n}\left(\vec{\Phi}_{d_{2}}\left(Z_{i}\right) \mathbb{1}_{Z_{i} \leq \sqrt{n}}-\mathbb{E}\left[\vec{\Phi}_{d_{2}}\left(Z_{i}\right) \mathbb{1}_{Z_{i} \leq \sqrt{n}}\right]\right)\right)\right\rangle_{2, d_{2}} \\
\zeta_{n}^{(2)}(\vec{t}) & :=\left\langle\vec{t}, \mathbf{G}_{d_{2}}^{-1}\left(\frac{1}{n} \sum_{i=1}^{n}\left(\vec{\Phi}_{d_{2}}\left(Z_{i}\right) \mathbb{1}_{Z_{i}>\sqrt{n}}-\mathbb{E}\left[\vec{\Phi}_{d_{2}}\left(Z_{i}\right) \mathbb{1}_{Z_{i}>\sqrt{n}}\right]\right)\right)\right\rangle_{2, d_{2}} .
\end{aligned}
$$

So we have the following inequality

$$
\left\|\widehat{S}_{X, \widehat{m}}-S_{X}\right\|^{2} \leq 3\left\|S_{X}-S_{X, m}\right\|^{2}+2 \operatorname{pen}_{2}(m)+16\left(\sup _{\vec{t} \in \mathcal{B}(m, \widehat{m})}\left(\zeta_{n}^{(1)}(\vec{t})\right)^{2}+\sup _{\vec{t} \in \mathcal{B}(m, \hat{m})}\left(\zeta_{n}^{(2)}(\vec{t})\right)^{2}\right)-2 \operatorname{pen}_{2}(\widehat{m}) .
$$

Now let $q$ be a function such that for any $m, m^{\prime}$, we have $: 4 q\left(m, m^{\prime}\right) \leq \operatorname{pen}_{2}(m)+\operatorname{pen}_{2}\left(m^{\prime}\right)$.

$$
\begin{aligned}
\left\|\widehat{S}_{X, \widehat{m}}-S_{X}\right\|^{2} \leq & 3\left\|S_{X}-S_{X, m}\right\|^{2}+4 \operatorname{pen}_{2}(m)+16\left[\sup _{\vec{t} \in \mathcal{B}(m, \hat{m})}\left(\zeta_{n}^{(1)}(\vec{t})\right)^{2}-q(m, \widehat{m})\right]_{+}+16 \sup _{\vec{t} \in \mathcal{B}(m, \widehat{m})}\left(\zeta_{n}^{(2)}(\vec{t})\right)^{2} \\
\leq & 3\left\|S_{X}-S_{X, m}\right\|^{2}+4 \operatorname{pen}_{2}(m)+16 \sum_{m^{\prime} \in \mathcal{M}_{n}^{(2)}}\left\{\sup _{\vec{t} \in \mathcal{B}\left(m, m^{\prime}\right)}\left(\zeta_{n}^{(1)}(\vec{t})\right)^{2}-q\left(m, m^{\prime}\right)\right\}_{+} \\
& +16 \sup _{\vec{t} \in \mathcal{B}(m, \widehat{m})}\left(\zeta_{n}^{(2)}(\vec{t})\right)^{2} .
\end{aligned}
$$

We now use the following result which ensures the validity of Theorem 4.3.

Proposition 7.3. Under the assumptions of Theorem 4.3, then there exists a universal constant $C>0$ such that for $q\left(m, m^{\prime}\right)=\kappa_{2} \varrho^{2}\left(\mathbf{G}_{m \vee m^{\prime}}^{-1}\right) \mathbb{E}\left[Z_{1}\right] \frac{\log n}{n}$

$$
\begin{aligned}
& \text { (i) } \mathbb{E}\left[\left\{\sup _{\vec{t} \in \mathcal{B}\left(m, m^{\prime}\right)}\left(\zeta_{n}^{(1)}(\vec{t})\right)^{2}-q\left(m, m^{\prime}\right)\right\}_{+}\right] \leq \frac{C}{n} \\
& \text { (ii) } \mathbb{E}\left[\sup _{\vec{t} \in \mathcal{B}(m, \hat{m})}\left(\zeta_{n}^{(2)}(\vec{t})\right)^{2}\right] \leq \frac{\mathbb{E}\left[Z_{1}^{3}\right]}{n}
\end{aligned}
$$

Finally, $\mathbb{E}\left\|\widehat{S}_{X, \widehat{m}}-S_{X}\right\|^{2} \leq 4 \inf _{m \in \mathcal{M}_{n}^{(2)}}\left\{\left\|S_{X}-S_{X, m}\right\|^{2}+\operatorname{pen}_{2}(m)\right\}+\frac{C}{n}$

Proof of Proposition 7.3. To prove $(i)$, we apply a Talagrand inequality. So we need to determine $H, M_{1}$ and $v$. 
- Let us start with the empirical process, first let us notice that

$$
\begin{aligned}
& \mathbb{E}\left[\sup _{\vec{t}_{m^{*}} \in \mathcal{B}\left(m, m^{\prime}\right)}\left|\zeta_{n}^{(1)}\left(\vec{t}_{m^{*}}\right)\right|^{2}\right] \\
& \quad=\mathbb{E}\left[\sup _{\vec{t}_{m^{*}} \in \mathcal{B}\left(m, m^{\prime}\right)}\left|\left\langle\vec{t}_{m^{*}}, \mathbf{G}_{d_{2}}^{-1}\left(\frac{1}{n} \sum_{i=1}^{n}\left(\vec{\Phi}_{d_{2}}\left(Z_{i}\right) \mathbb{1}_{Z_{i} \leq \sqrt{n}}-\mathbb{E}\left[\vec{\Phi}_{d_{2}}\left(Z_{i}\right) \mathbb{1}_{Z_{i} \leq \sqrt{n}}\right]\right)\right)\right\rangle_{2, d_{2}}\right|^{2}\right] \\
& \quad=\mathbb{E}\left[\sup _{\vec{t}_{m^{*}} \in \mathcal{B}\left(m, m^{\prime}\right)}\left|\left\langle\vec{t}_{m^{*}}, \mathbf{G}_{m^{*}}^{-1}\left(\frac{1}{n} \sum_{i=1}^{n}\left(\vec{\Phi}_{m^{*}}\left(Z_{i}\right) \mathbb{1}_{Z_{i} \leq \sqrt{n}}-\mathbb{E}\left[\vec{\Phi}_{m^{*}}\left(Z_{i}\right) \mathbb{1}_{Z_{i} \leq \sqrt{n}}\right]\right)\right)\right\rangle_{2, m^{*}}\right|^{2}\right] .
\end{aligned}
$$

We now apply Cauchy-Schwarz inequality and get

$$
\begin{aligned}
& \mathbb{E}\left[\sup _{\vec{t}_{m^{*}} \in \mathcal{B}\left(m, m^{\prime}\right)}\left|\zeta_{n}^{(1)}\left(\vec{t}_{m^{*}}\right)\right|^{2}\right] \\
& \leq \mathbb{E}\left[\sup _{\vec{t}_{m^{*}} \in \mathcal{B}\left(m, m^{\prime}\right)}\left\|\vec{t}_{m^{*}}\right\|_{2, m^{*}}^{2}\left\|\mathbf{G}_{m^{*}}^{-1}\left(\frac{1}{n} \sum_{i=1}^{n}\left(\vec{\Phi}_{m^{*}}\left(Z_{i}\right) \mathbb{1}_{Z_{i} \leq \sqrt{n}}-\mathbb{E}\left[\vec{\Phi}_{m^{*}}\left(Z_{i}\right) \mathbb{1}_{Z_{i} \leq \sqrt{n}}\right]\right)\right)\right\|_{2, m^{*}}^{2}\right] \\
& \leq \mathbb{E}\left[\left\|\mathbf{G}_{m^{*}}^{-1}\left(\frac{1}{n} \sum_{i=1}^{n}\left(\vec{\Phi}_{m^{*}}\left(Z_{i}\right) \mathbb{1}_{Z_{i} \leq \sqrt{n}}-\mathbb{E}\left[\vec{\Phi}_{m^{*}}\left(Z_{i}\right) \mathbb{1}_{Z_{i} \leq \sqrt{n}}\right]\right)\right)\right\|_{2, m^{*}}\right] .
\end{aligned}
$$

It follows that, with Inequality (25)

$$
\begin{aligned}
& \mathbb{E}\left[\sup _{\vec{t}_{m^{*}} \in \mathcal{B}\left(m, m^{\prime}\right)}\left|\zeta_{n}^{(1)}\left(\vec{t}_{m^{*}}\right)\right|^{2}\right] \\
& \quad \leq \varrho^{2}\left(\mathbf{G}_{m^{*}}^{-1}\right) \mathbb{E}\left[\sum_{j=0}^{m^{*}-1}\left(\frac{1}{n} \sum_{i=1}^{n}\left(\Phi_{j}\left(Z_{i}\right) \mathbb{1}_{Z_{i} \leq \sqrt{n}}-\mathbb{E}\left[\Phi_{j}\left(Z_{i}\right) \mathbb{1}_{Z_{i} \leq \sqrt{n}}\right]\right)\right)^{2}\right] \\
& \quad \leq \varrho^{2}\left(\mathbf{G}_{m^{*}}^{-1}\right) \sum_{j=0}^{m^{*}-1} \operatorname{Var}\left(\Phi_{j}\left(Z_{1}\right) \mathbb{1}_{Z_{1} \leq \sqrt{n}}\right) \leq \frac{\varrho^{2}\left(\mathbf{G}_{m^{*}}^{-1}\right)}{n} \sum_{j=0}^{m^{*}-1} \mathbb{E}\left[\Phi_{j}^{2}\left(Z_{1}\right) \mathbb{1}_{Z_{1} \leq \sqrt{n}}\right] \leq \frac{\varrho^{2}\left(\mathbf{G}_{m^{*}}^{-1}\right)}{n} \mathbb{E}\left[Z_{1} \mathbb{1}_{Z_{1} \leq \sqrt{n}}\right] .
\end{aligned}
$$

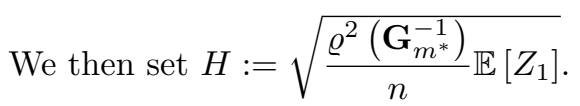

- Now for the term of variance, let $\vec{t}_{m^{*}} \in \mathcal{B}\left(m, m^{\prime}\right)$

$$
\begin{aligned}
\mathbb{E} & {\left[\mid\left\langle\vec{t}_{m^{*}}, \mathbf{G}_{d_{2}}^{-1} \vec{\Phi}_{d_{2}}\left(Z_{1}\right) \mathbb{1}_{\left.\left.Z_{1} \leq \sqrt{n}\right\rangle\left._{2, d_{2}}\right|^{2}\right]}\right.\right.} \\
& =\mathbb{E}\left[\left|\left\langle\vec{t}_{m^{*}}, \mathbf{G}_{m^{*}}^{-1} \vec{\Phi}_{m^{*}}\left(Z_{1}\right) \mathbb{1}_{Z_{1} \leq \sqrt{n}}\right\rangle_{2, m^{*}}\right|^{2}\right] \leq \mathbb{E}\left[\sup _{\vec{t}_{m^{*}} \in \mathcal{B}\left(m, m^{\prime}\right)}\left\|\vec{t}_{m^{*}}\right\|_{2, m^{*}}^{2}\left\|\mathbf{G}_{m^{*}}^{-1} \vec{\Phi}_{m^{*}}\left(Z_{1}\right) \mathbb{1}_{Z_{1} \leq \sqrt{n}}\right\|_{2, m^{*}}^{2}\right] \\
& \leq \mathbb{E}\left[\left\|\mathbf{G}_{m^{*}}^{-1} \vec{\Phi}_{m^{*}}\left(Z_{1}\right) \mathbb{1}_{Z_{1} \leq \sqrt{n}}\right\|_{2, m^{*}}^{2}\right] \leq \varrho^{2}\left(\mathbf{G}_{m^{*}}^{-1}\right) \mathbb{E}\left[Z_{1} \mathbb{1}_{Z_{1} \leq \sqrt{n}}\right] .
\end{aligned}
$$

So we set $v:=\mathbb{E}\left[Z_{1}\right] \varrho^{2}\left(\mathbf{G}_{m^{*}}^{-1}\right)$.

- First notice again

$$
\sup _{\vec{t}_{m^{*}} \in \mathcal{B}\left(m, m^{\prime}\right)} \sup _{x \in \mathbb{R}^{+}}\left\langle\vec{t}_{m^{*}}, \mathbf{G}_{d_{2}}^{-1} \vec{\Phi}_{d_{2}}(x) \mathbb{1}_{x \leq \sqrt{n}}\right\rangle_{2, d_{2}}=\sup _{\vec{t}_{m^{*}} \in \mathcal{B}\left(m, m^{\prime}\right)} \sup _{x \in \mathbb{R}^{+}}\left\langle\vec{t}_{m^{*}}, \mathbf{G}_{m^{*}}^{-1} \vec{\Phi}_{m^{*}}(x) \mathbb{1}_{x \leq \sqrt{n}}\right\rangle_{2, m^{*}}
$$


Now applying Cauchy-Schwarz inequality and using Inequality 25 again

$$
\begin{aligned}
& \quad \sup _{\vec{t}_{m^{*}} \in \mathcal{B}\left(m, m^{\prime}\right)} \|\left\langle\vec{t}_{m^{*}}, \mathbf{G}_{d_{2}}^{-1} \vec{\Phi}_{d_{2}}(x) \mathbb{1}_{x \leq \sqrt{n}\rangle_{2, d_{2}} \|_{\infty}}\right. \\
& \leq \sup _{x \in \mathbb{R}^{+}}\left\|\mathbf{G}_{m^{*}}^{-1} \vec{\Phi}_{m^{*}}(x) \mathbb{1}_{x \leq n}\right\|_{2, m^{*}} \leq \sup _{x \in \mathbb{R}^{+}} \sqrt{t \vec{\Phi}_{m^{*}}(x)^{t} \mathbf{G}_{m^{*}}^{-1} \mathbf{G}_{m^{*}}^{-1} \vec{\Phi}_{m^{*}}(x) \mathbb{1}_{x \leq \sqrt{n}}} \\
& \leq \sqrt{\varrho^{2}\left(\mathbf{G}_{m^{*}}^{-1}\right) \sup _{x \in \mathbb{R}^{+}} \sum_{j=0}^{m^{*}-1} \Phi_{j}^{2}(x) \mathbb{1}_{x \leq \sqrt{n}} \leq \sqrt{\sqrt{n} \varrho^{2}\left(\mathbf{G}_{m^{*}}^{-1}\right)} .}
\end{aligned}
$$

We take $M_{1}=\sqrt{\sqrt{n} \varrho^{2}\left(\mathbf{G}_{m^{*}}^{-1}\right)}$.

We apply Talagrand's inequality for $\xi^{2}=\frac{2}{K_{1}} \log (n)$. We get

$$
\begin{aligned}
& \mathbb{E}\left[\left\{\sup _{\vec{t} \in \mathcal{B}\left(m, m^{\prime}\right)}\left|\zeta_{n}^{(1)}(\vec{t})\right|^{2}-\kappa_{3} \varrho^{2}\left(\mathbf{G}_{m^{*}}^{-1}\right) \mathbb{E}\left[Z_{1}\right] \frac{\log n}{n}\right\}_{+}\right] \\
& \leq \frac{4}{K_{1}} \frac{\mathbb{E}\left[Z_{1}\right] \varrho^{2}\left(\mathbf{G}_{m^{*}}^{-1}\right)}{n} e^{-K_{1} \xi^{2}}+\frac{98 n \varrho^{2}\left(\mathbf{G}_{m^{*}}^{-1}\right)}{K_{1} n^{2} C^{2}\left(\xi^{2}\right)} e^{-\frac{2 K_{1} C(\xi) \xi}{7 \sqrt{2}} \sqrt{\mathbb{E}\left[Z_{1}\right]} n^{1 / 4}}
\end{aligned}
$$

which implies that

$$
\begin{aligned}
& \sum_{m^{\prime} \in \mathcal{M}_{n}^{(2)}} \mathbb{E}\left[\left\{\sup _{\vec{t} \in \mathcal{B}\left(m, m^{\prime}\right)}\left|\zeta_{n}^{(1)}(\vec{t})\right|^{2}-\kappa_{3} \varrho^{2}\left(\mathbf{G}_{m^{*}}^{-1}\right) \mathbb{E}\left[Z_{1}\right] \frac{\log n}{n}\right\}_{+}\right] \\
& \leq \sum_{m^{\prime} \in \mathcal{M}_{n}^{(2)}} \frac{4}{K_{1}} \frac{\mathbb{E}\left[Z_{1}\right] \varrho^{2}\left(\mathbf{G}_{m^{*}}^{-1}\right)}{n} e^{-K_{1} \xi^{2}}+\frac{98 \sqrt{n} \varrho^{2}\left(\mathbf{G}_{m^{*}}^{-1}\right)}{K_{1} n^{2} C^{2}\left(\xi^{2}\right)} e^{-\frac{2 K_{1} C(\xi) \xi}{7 \sqrt{2}} \sqrt{\mathbb{E}\left[Z_{1}\right]} n^{1 / 4}} .
\end{aligned}
$$

Thus we have under Assumption (B2)

$$
\sum_{m^{\prime} \in \mathcal{M}_{n}^{(2)}} \frac{\varrho^{2}\left(\mathbf{G}_{m^{*}}^{-1}\right)}{n} e^{-K_{1} \xi^{2}} \leq \sum_{m^{\prime} \in \mathcal{M}_{n}^{(2)}} \frac{\varrho^{2}\left(\mathbf{G}_{m^{*}}^{-1}\right)}{n} \frac{1}{n^{2}} \leq \frac{\left|\mathcal{M}_{n}^{(2)}\right|}{n^{2}} \leq \frac{1}{n} .
$$

And

$$
\sum_{m^{\prime} \in \mathcal{M}_{n}^{(2)}} \frac{\sqrt{n} \varrho^{2}\left(\mathbf{G}_{m^{*}}^{-1}\right)}{K_{1} n^{2} C^{2}\left(\xi^{2}\right)} e^{-\frac{2 K_{1} C(\xi) \xi}{7 \sqrt{2}} \sqrt{\mathbb{E}\left[Z_{1}\right]} n^{1 / 4}} \leq \sum_{m^{\prime} \in \mathcal{M}_{n}^{(2)}} \frac{C \sqrt{n}}{(\log n)^{2} n} e^{-\frac{2 K_{1} C(\xi) \xi}{7 \sqrt{2}} \sqrt{\mathbb{E}\left[Z_{1}\right]} n^{1 / 4}} \leq \frac{C}{n}
$$

So for $q\left(m, m^{\prime}\right)=\kappa_{3} \varrho^{2}\left(\mathbf{G}_{m^{*}}^{-1}\right) \mathbb{E}\left[Z_{1}\right] \frac{\log n}{n}$ we just showed that

$$
\sum_{m^{\prime} \in \mathcal{M}_{n}^{(2)}} \mathbb{E}\left[\left\{\sup _{\vec{t} \in \mathcal{B}\left(m, m^{\prime}\right)}\left|\zeta_{n}^{(1)}(\vec{t})\right|^{2}-\kappa_{3} \varrho^{2}\left(\mathbf{G}_{m^{*}}^{-1}\right) \mathbb{E}\left[Z_{1}\right] \frac{\log n}{n}\right\}_{+}\right] \leq \frac{C}{n}
$$

Now we prove $(i i)$. We have, using (A2),

$$
\begin{aligned}
\mathbb{E}\left[\sup _{\vec{t} \in \mathcal{B}(m, \hat{m})}\left|\zeta_{n}^{(2)}\left(\vec{t}_{m^{*}}\right)\right|^{2}\right] & =\mathbb{E}\left[\sup _{\vec{t} \in \mathcal{B}\left(d_{2}, d_{2}\right)}\left|\left\langle\vec{t}, \mathbf{G}_{d_{2}}^{-1}\left(\frac{1}{n} \sum_{i=1}^{n}\left(\vec{\Phi}_{d_{2}}\left(Z_{i}\right) \mathbb{1}_{Z_{i}>\sqrt{n}}-\mathbb{E}\left[\vec{\Phi}_{d_{2}}\left(Z_{i}\right) \mathbb{1}_{Z_{i}>\sqrt{n}}\right]\right)\right)\right\rangle_{2, d_{2}}\right|^{2}\right] \\
& \leq \frac{\varrho^{2}\left(\mathbf{G}_{d_{2}}^{-1}\right)}{n} \mathbb{E}\left[Z_{1} \mathbb{1}_{Z_{1}>\sqrt{n}}\right] \leq \frac{\mathbb{E}\left[Z_{1}^{3}\right]}{n} .
\end{aligned}
$$

In the end we have the desired result.

Proof of Corollary 4.4. The beginning of the proof is the same as the proof of Theorem 4.3 except that we consider $\widetilde{m}_{2}$ (defined by Equation (23)) instead of $\widehat{m}_{2}$ and $\widehat{p e n}_{2}$ (defined by Equation (24)) instead of pen 2 . 
Starting from Equation (29) we have

$$
\begin{gathered}
\left\|\widehat{S}_{X, \widehat{m}}-S_{X}\right\|^{2} \leq 3\left\|S_{X}-S_{X, m}\right\|^{2}+2 \widehat{\operatorname{pen}}_{2}(m)+16\left(\sup _{\vec{t} \in \mathcal{B}(m, \hat{m})}\left(\zeta_{n}^{(1)}(\vec{t})\right)^{2}+\sup _{\vec{t} \in \mathcal{B}(m, \hat{m})}\left(\zeta_{n}^{(2)}(\vec{t})^{2}\right)-2 \widehat{\operatorname{pen}}_{2}\left(\widetilde{m}_{2}\right)\right. \\
\leq 3\left\|S_{X}-S_{X, m}\right\|^{2}+2 \widehat{\operatorname{pen}}_{2}(m)+16\left(\sup _{\vec{t} \in \mathcal{B}(m, \hat{m})}\left(\zeta_{n}^{(1)}(\vec{t})\right)^{2}+\sup _{\vec{t} \in \mathcal{B}(m, \hat{m})}\left(\zeta_{n}^{(2)}(\vec{t})\right)^{2}\right) \\
\quad-2 \operatorname{pen}_{2}\left(\widetilde{m}_{2}\right)+2 \operatorname{pen}_{2}\left(\widetilde{m}_{2}\right)-2 \widehat{\operatorname{pen}}_{2}\left(\widetilde{m}_{2}\right) \\
\leq 3\left\|S_{X}-S_{X, m}\right\|^{2}+2 \widehat{\operatorname{pen}}_{2}(m)+16\left\{\underset{\vec{t} \in \mathcal{B}(m, \hat{m})}{\sup }\left(\zeta_{n}^{(1)}(\vec{t})\right)^{2}-q\left(m, \widetilde{m}_{2}\right)\right\}_{+}+2 \operatorname{pen}_{2}(m) \\
+16 \sup _{\vec{t} \in \mathcal{B}(m, \hat{m})}\left(\zeta_{n}^{(2)}(\vec{t})\right)^{2}+2\left\{\operatorname{pen}_{2}\left(\widetilde{m}_{2}\right)-\widehat{\operatorname{pen}}_{2}\left(\widetilde{m}_{2}\right)\right\}_{+} \cdot
\end{gathered}
$$

We now apply the following Proposition which ensures the validity of Corollary 4.4

Proposition 7.4. Under the Assumptions of Corollary 4.4, the following holds

$$
\mathbb{E}\left[\widehat{\operatorname{pen}}_{2}(m)\right]=2 \operatorname{pen}_{2}(m) \text { and } \mathbb{E}\left[\left\{\operatorname{pen}_{2}\left(\widetilde{m}_{2}\right)-\widehat{\operatorname{pen}}_{2}\left(\widetilde{m}_{2}\right)\right\}_{+}\right] \leq \frac{C}{n} \text {. }
$$

Finally, $\mathbb{E}\left\|S_{X}-\widehat{S}_{X, \widetilde{m}_{2}}\right\|^{2} \leq \inf _{m \in \mathcal{M}_{n}^{(2)}}\left\{\left\|S_{X}-S_{X, m}\right\|^{2}+\operatorname{pen}_{2}(m)\right\}+\frac{C}{n}$.

Proof of Proposition 7.4. First let us notice the following

$$
\mathbb{E}\left[\widehat{\operatorname{pen}}_{2}(m)\right]=2 \kappa_{2} \mathbb{E}\left[\bar{Z}_{n}\right] \frac{\varrho^{2}\left(\mathbf{G}_{m}^{-1}\right) \log n}{n}=2 \kappa_{2} \mathbb{E}\left[Z_{1}\right] \frac{\varrho^{2}\left(\mathbf{G}_{m}^{-1}\right) \log n}{n}=2 \operatorname{pen}_{2}(m) .
$$

For the second inequality, let us introduce the following favorable set: $\Lambda=\left\{\left|\mathbb{E}\left[Z_{1}\right]-\bar{Z}_{n}\right| \leq \mathbb{E}\left[Z_{1}\right] / 2\right\}$, which yields

$$
\begin{aligned}
& \mathbb{E}\left[\left\{\operatorname{pen}_{2}\left(\widetilde{m}_{2}\right)-\widehat{\operatorname{pen}}_{2}\left(\widetilde{m}_{2}\right)\right\}_{+}\right]=\mathbb{E} {\left[\left\{2 \kappa_{2}\left(\frac{\mathbb{E}\left[Z_{1}\right]}{2}-\bar{Z}_{n}\right) \varrho^{2}\left(\mathbf{G}_{\widetilde{m}_{2}}^{-1}\right) \frac{\log n}{n}\right\}_{+} \mathbb{1}_{\Lambda}\right] } \\
&+\mathbb{E}\left[\left\{2 \kappa_{2}\left(\frac{\mathbb{E}\left[Z_{1}\right]}{2}-\bar{Z}_{n}\right) \varrho^{2}\left(\mathbf{G}_{\widetilde{m}_{2}}^{-1}\right) \frac{\log n}{n}\right\}_{+} \mathbb{1}_{\Lambda^{c}}\right] .
\end{aligned}
$$

Yet on the set $\Lambda, \mathbb{E}\left[Z_{1}\right] / 2-\bar{Z}_{n} \leq 0$ which yields

$$
\begin{aligned}
\mathbb{E}\left[\left\{\operatorname{pen}_{2}\left(\widetilde{m}_{2}\right)-\widehat{\operatorname{pen}}_{2}\left(\widetilde{m}_{2}\right)\right\}_{+}\right] & =\mathbb{E}\left[2 \kappa_{2}\left(\frac{\mathbb{E}\left[Z_{1}\right]}{2}-\bar{Z}_{n}\right) \varrho^{2}\left(\mathbf{G}_{\widetilde{m}_{2}}^{-1}\right) \mathbb{1}_{\Lambda^{c}}\right] \frac{\log n}{n} \\
& \leq \mathbb{E}\left[2 \kappa_{2}\left|\mathbb{E}\left[Z_{1}\right]-\bar{Z}_{n}\right| \varrho^{2}\left(\mathbf{G}_{\widetilde{m}_{2}}^{-1}\right) \mathbb{1}_{\Lambda^{c}}\right] \frac{\log n}{n} .
\end{aligned}
$$

Now we apply Cauchy-Schwarz

$$
\mathbb{E}\left[\left|\mathbb{E}\left[Z_{1}\right]-\bar{Z}_{n}\right| \mathbb{1}_{\Lambda^{c}}\right] \leq \sqrt{\mathbb{E}\left|\mathbb{E}\left[Z_{1}\right]-\bar{Z}_{n}\right|^{2}} \sqrt{\mathbb{P}\left[\Lambda^{c}\right]}=\sqrt{\operatorname{Var}\left[\bar{Z}_{n}\right]} \sqrt{\mathbb{P}\left[\left|\mathbb{E}\left[Z_{1}\right]-\bar{Z}_{n}\right| \geq \frac{\mathbb{E}\left[Z_{1}\right]}{2}\right]}
$$

We apply Markov inequality then Rosenthal inequality

$$
\mathbb{E}\left[\left|\mathbb{E}\left[Z_{1}\right]-\bar{Z}_{n}\right| \mathbb{1}_{\Lambda^{c}}\right] \leq \frac{\sqrt{\operatorname{Var}\left[Z_{1}\right]}}{\sqrt{n}} \sqrt{\frac{\mathbb{E}\left[\left|\mathbb{E}\left[Z_{1}\right]-\bar{Z}_{n}\right|^{2}\right]}{\mathbb{E}\left[Z_{1}\right]^{2}}} \leq \frac{\sqrt{\operatorname{Var}\left[Z_{1}\right]}}{\mathbb{E}\left[Z_{1}\right] n} .
$$

Moreover under Assumption (B2)

$$
\mathbb{E}\left[\left\{\operatorname{pen}_{2}\left(\widetilde{m}_{2}\right)-\widehat{\operatorname{pen}}_{2}\left(\widetilde{m}_{2}\right)\right\}_{+}\right] \leq \frac{C}{n} \mathbb{E}\left[\varrho^{2}\left(\mathbf{G}_{\widetilde{m}_{2}}^{-1}\right) \frac{\log n}{n}\right] \leq \frac{C^{\prime}}{n}
$$

which ends the proof. 
Appendix A.

Lemma A.1. (Talagrand's inequality) Let $Y_{1}, \ldots, Y_{n}$ be i.i.d. variables and

$$
r_{n}(f)=\frac{1}{n} \sum_{k=1}^{n}\left(f\left(Y_{k}\right)-\mathbb{E}\left[f\left(Y_{k}\right)\right]\right)
$$

for $f$ belonging to some countable set $\mathcal{F}$ of uniformly bounded measurable functions. Then for $\xi^{2}>0$,

$$
\mathbb{E}\left[\left\{\sup _{f \in \mathcal{F}}\left|r_{n}(f)\right|^{2}-2\left(1+2 \xi^{2}\right) H^{2}\right\}_{+}\right] \leq \frac{4}{K_{1}}\left(\frac{v}{n} e^{-K_{1} \xi^{2} \frac{n H^{2}}{v}}+\frac{98 M_{1}^{2}}{K_{1} n^{2} C^{2}\left(\xi^{2}\right)} e^{-\frac{2 K_{1} C(\xi) \xi}{7 \sqrt{2}} \frac{n H}{M_{1}}}\right)
$$

with constants $C(\xi)=\left(\sqrt{1+\xi^{2}}-1\right) \wedge 1$ and $K_{1}=\frac{1}{6}, M_{1}, H$ and $v$ are such that

$$
\sup _{f \in \mathcal{F}}\|f\|_{\infty} \leq M_{1}, \quad \mathbb{E}\left[\sup _{f \in \mathcal{F}}\left|r_{n}(f)\right|\right] \leq H, \quad \sup _{f \in \mathcal{F}} \operatorname{Var}\left(f\left(Y_{1}\right)\right) \leq v .
$$

\section{Appendix B.}

$$
\begin{aligned}
\widetilde{S}_{X, m}(x) & =\sum_{k=0}^{m-1} \hat{b}_{k}(f) \tilde{\Phi}_{k}(x)=\sum_{k=0}^{m-1} \int_{x}^{+\infty} \hat{b}_{k}(f) \varphi_{k}(u) \mathrm{d} u \\
& =\sqrt{2} \sum_{k=0}^{m-1} \hat{b}_{k}(f) \sum_{j=0}^{k}\left(\begin{array}{c}
k \\
j
\end{array}\right) \frac{(-2)^{j}}{j !} \int_{x}^{+\infty} e^{-u} u^{j} \mathrm{~d} u=\sqrt{2} \sum_{k=0}^{m-1} \hat{b}_{k}(f) \sum_{j=0}^{k}\left(\begin{array}{c}
k \\
j
\end{array}\right) \frac{(-2)^{j}}{j !} \Gamma(j+1, x) .
\end{aligned}
$$

where $\Gamma(.,$.$) is the upper incomplete gamma function defined in Abramowitz and Stegun (1964). Then the$ incomplete gamma function can be decomposed again in the Laguerre basis. First recall

$$
\Gamma(j+1, x)=j ! e^{-x} \sum_{i=0}^{j} \frac{x^{i}}{i !} \quad \text { and } \quad \frac{x^{i}}{i !}=\sum_{\ell=0}^{i}(-1)^{\ell}\left(\begin{array}{c}
i \\
i-\ell
\end{array}\right) L_{\ell}(x)
$$

which yields

$$
\Gamma(j+1, x)=j ! e^{-x} \sum_{i=0}^{j} 2^{-j} \sum_{\ell=0}^{i}(-1)^{\ell}\left(\begin{array}{c}
i \\
i-\ell
\end{array}\right) L_{\ell}(2 x)=\frac{j !}{\sqrt{2}} \sum_{i=0}^{j} 2^{-j} \sum_{\ell=0}^{i}(-1)^{\ell}\left(\begin{array}{c}
i \\
i-\ell
\end{array}\right) \varphi_{\ell}(x) .
$$

Thus

$$
\begin{aligned}
\widetilde{S}_{X, m}(x) & =\sum_{k=0}^{m-1} \hat{b}_{k}(f) \sum_{j=0}^{k}\left(\begin{array}{c}
k \\
j
\end{array}\right) \frac{(-2)^{j}}{j !} j ! \sum_{i=0}^{j} 2^{-j} \sum_{\ell=0}^{i}(-1)^{\ell}\left(\begin{array}{c}
i \\
i-\ell
\end{array}\right) \varphi_{\ell}(x) \\
& =\sum_{k=0}^{m-1} \sum_{j=0}^{k} \sum_{i=0}^{j} \sum_{\ell=0}^{i} \hat{b}_{k}(f)\left(\begin{array}{c}
k \\
j
\end{array}\right)(-1)^{j+\ell}\left(\begin{array}{c}
i \\
i-\ell
\end{array}\right) \varphi_{\ell}(x) .
\end{aligned}
$$

The difficulty is to write $\widetilde{S}_{X, m}(x)=\sum_{k=0}^{m-1} \tilde{b}_{k}(f) \varphi_{k}(x)$.

\section{ACKNOWLEDGEMENTS}

The author would like to thank Fabienne Comte for her fruitful advice and suggestions and Valentine Genon-Catalot for her interest in this work.

\section{REFERENCES}

Abramowitz, M. and Stegun, I. A. (1964). Handbook of Mathematical Functions with Formulas, Graphs, and Mathematical Tables. Dover, New York, ninth dover printing, tenth gpo printing edition.

Bibinger, M., Jirak, M., and Reiß, M. (2014). Improved volatility estimation based on limit order books. Preprint, arXiv:1408.3768.

Birgé, L. (1999). An alternative point of view on Lepski's method. In State of the art in probability and statistics: Festschrift for Willem R. van Zwet, pages 113-133. IMS Lecture Notes-Monograph Series.

Birgé, L. and Massart, P. (1997). From model selection to adaptive estimation. In Festschrift for Lucien Le Cam, pages 55-87. Springer. 
Bongioanni, B. and Torrea, J. L. (2009). What is a Sobolev space for the Laguerre function systems? Studia Math., 192(2):147-172.

Butucea, C. (2004). Deconvolution of supersmooth densities with smooth noise. The Canadian Journal of Statistics, 32(2):181-192.

Butucea, C. and Tsybakov, A. (2008a). Sharp optimality in density deconvolution with dominating bias I. Theory Proba. Appl., 52(1):24-39.

Butucea, C. and Tsybakov, A. (2008b). Sharp optimality in density deconvolution with dominating bias II. Theory Proba. Appl., 52(2):237-249.

Carroll, R. J. and Hall, P. (1988). Optimal rates of convergence for deconvolving a density. Journal of the American Statistical Association, 83(404):1184-1186.

Comte, F., Cuenod, C.-A., Pensky, M., and Rozenholc, Y. (2013). Laplace deconvolution and its application to dynamic contrast enhanced imaging. Prépublication MAP5 2012-17.

Comte, F. and Genon-Catalot, V. (2013). Adaptive Laguerre density estimation for mixed Poisson models. Preprint, hal-00848158.

Comte, F. and Lacour, C. (2011). Data-driven density estimation in the presence of additive noise with unknown distribution. Journal of the Royal Statistical Society: Series B, 73:601-627.

Comte, F., Rozenholc, Y., and Taupin, M.-L. (2006). Penalized contrast estimator for adaptive density deconvolution. The Canadian Journal of Statistics, 3(34):431-452.

Dattner, I., Goldenshluger, A., and Juditsky, A. (2011). On deconvolution of distribution functions. The Annals of Statistics, 39(5):2477-2501.

Dattner, I. and Reiser, B. (2013). Estimation of distribution functions in measurement error models. Journal of Statistical Planning and Inference, 143(3):479 - 493.

Delaigle, A. and Gijbels, I. (2004). Bootstrap bandwidth selection in kernel density estimation from a contaminated sample. Annals of the Institute of Statistical Mathematics, 56(1):19-47.

Devroye, L. (1987). A Course in Density Estimation. In Birkhauser:Boston MA.

Efromovich, S. (1997). Density estimation for the case of supersmooth measurement errors. Journal of the American Statistical Association, 92:526-535.

Fan, J. (1991). On the optimal rates of convergence for nonparametric deconvolution problems. The Annals of Statistics, 19(3):1257-1272.

Guerre, E., Perrigne, I., and Vuong, Q. (2000). Optimal nonparametric estimation of first-price auctions. Econometrica, 68(3):525-574.

Hall, P. and Lahiri, S. N. (2008). Estimation of distributions, moments and quantiles in deconvolution problems. The Annals of Statistics, 36(5):2110-2134.

Jirak, M., Meister, A., and Reiß, M. (2014). Adaptive function estimation in nonparametric regression with one-sided errors. The Annals of Statistics, 42(5):1970-2002.

Johannes, J. (2009). Deconvolution with unknown error distribution. The Annals of Statistics, 37(5A):23012323.

Juditsky, A. and Lambert-Lacroix, S. (2004). On minimax density estimation on $\mathbb{R}$. Bernoulli, 10(2):187220.

Kappus, J. and Mabon, G. (2013). Adaptive density estimation in deconvolution problems with unknown error distribution. Preprint, hal-00915982.

Massart, P. (2003). Concentration Inequalities and Model selection, volume 1896 of Lecture Notes in Mathematics. Springer, Berlin. Lectures from the 33rd Summer School on Probability Theory bheld in SaintFlour, July 6-23, 2003, With a foreword by Jean Picard.

Meister, A. (2009). Deconvolution Problems in Nonparametric Statistics, volume 193 of Lecture Notes in Statistics. Springer-Verlag, Berlin.

Neumann, M. H. (1997). On the effect of estimating the error density in nonparametric deconvolution. Journal of Nonparametric Statistics, 7(4):307-330.

Pensky, M. and Vidakovic, B. (1999). Adaptive wavelet estimator for nonparametric density deconvolution. The Annals of Statistics, 27(6):2033-2053.

Reiß, M. and Selk, L. (2013). Estimating nonparametric functionals efficiently under one-sided errors. Preprint, arXiv:1407.4229.

Silverman, B. W. (1986). Density estimation for statistics and data analysis. Monographs on statistics and applied probability. Chapman and Hall Boca Raton, London, Glasgow, Weinheim.

Stefanski, L. (1990). Rates of convergence of some estimators in a class of deconvolution problems. Statistics and Probability Letters, 9:229-235.

Stefanski, S. and Carroll, R. (1990). Deconvoluting kernel density estimators. Statistics, 21:169-184. 
Vareschi, T. (2014). Noisy Laplace deconvolution with error in the operator. To appear in Journal of Statistical Planning and Inference.

Zhang, C.-H. (1990). Fourier methods for estimating mixing densities and distributions. The Annals of Statistics, 18(2):806-831. 